\title{
Genome-wide Analysis and Functional Characterization of the Della Gene Family Associated With Stress Tolerance in B. Napus
}

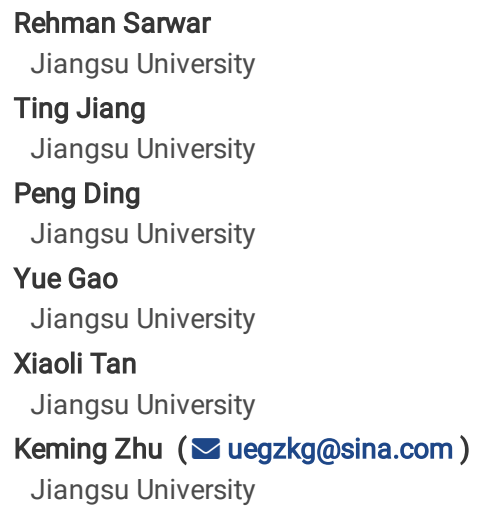

Research Article

Keywords: Brassica napus, gibberellins, DELLA, miRNA, RT-qPCR, abiotic stress, Sclerotinia sclerotiorum, genome-wide

Posted Date: March 1st, 2021

DOI: https://doi.org/10.21203/rs.3.rs-265062/v1

License: (c) (1) This work is licensed under a Creative Commons Attribution 4.0 International License. Read Full License 


\section{Abstract}

Background: Brassica napus is an essential crop for oil and livestock feed. Eventually, this crop's economic interest is at the most risk due to anthropogenic climate change. DELLA proteins constitute a significant repressor of plant growth to facilitate survival under constant stress conditions. DELLA proteins lack DNA binding domain, but can interact with various transcription factors or transcription regulators of different hormonal families. Significant progress has been made on Arabidopsis and cereal plants. However, no comprehensive study regarding DELLA proteins have been delineated in $B$. napus.

Results: In our study, we have identified 10 BnDELLA genes. All of the BnDELLAs are closely related to five AtDELLA genes, suggesting a relative function and structure. Chromosomal mapping revealed the uneven distribution of BnDELLAs on eight chromosomes, and site-specific selection assessment proposes $B n D E L L A s$ purifying selection. The motifs composition in all BnDELLA genes is uneven; however, every BnDELLA gene contains 12 highly conserved motifs, encoding DELLA and GRAS domain. The two known miRNAs (bna-miR6029 and bna-miR603) targets, BnC07RGA and BnA09GAl, were also predicted. Furthermore, quantitative real-time PCR (qRT-PCR) analysis has exhibited the BnDELLA genes' diverse expression patterns in various plant organs. Additionally, cis-acting element prediction shows that all BnDELLA genes contain light, stress, and hormone-responsive elements on their promoters. The gene ontology (GO) enrichment report indicated that BnDELLA genes mainly regulate stress responses. Through publicly available RNA-seq analysis, we determined the induced expression of BnDELLA genes by different biotic and abiotic stress treatments.

Conclusion: The present study provides essential information on the BnDELLA gene family in $B$. napus, illustrates the significance of BnDELLAs in stress adaptation, and suggests modulating BnDELLAs expression is a promising way to intensify $B$. napus stress tolerance and harvest index.

\section{Background}

Brassica napus is becoming the world's most economically important crop since the 1970s [1]. Significant progress has been made in the advancement of this crop selective breeding. The genetic alteration resulted in an improved $40 \%$ of high-quality vegetable oil, which has been used in the cooking and pharmaceutical application with the feed after oil isolation utilized as animal fodder. Unfortunately, B. napus horticulture is under constant pressure by anthropogenic climate change. This has led to severe loss in harvest index and oil production in many regions of the world and scarce its geographical distribution [2, 3]. Consequently, the effects of environmental stresses on B. napus will eventually lose their economic significance. Therefore, there is an imperative need to outline and specify critical components that can accommodate crops to the expected climate conditions.

Plants as sessile organisms evolved varied strategies to modulate their physiology to cope with fluctuating environmental conditions, such as higher salinity, drought, high/low temperature, and pathogen infections [4]. Tremendous work has been done to understand the role of plants' biochemical, molecular, and cellular response to these abiotic and biotic stresses $[5,6]$. These studies suggest that phytohormones mediate the plants' internal and external response to facilitate plant vitality under stress conditions. Among these hormones, Gibberellins (GAs) are considered one of the most vital hormones that dramatically affect plant physiology by cross-talking with multiple hormones [7, 8]. However, under external pressure, plants maintain GAs homeostasis by a family of coregulator DELLAs, which interact with a wide variety of transcriptional factors and transcriptional regulators of multiple hormones, to inhibit growth in allocating resources for plant survival $[9,10]$. Previously, it proposed that mutation in 17 amino acids of the DELLA N-terminal region resulted in severe dwarf transgenic plant gai-1 with dark green leaves, much like GA-deficient mutant ga 1-3 [11, 12]. Later it was demonstrated that the N-terminal region of DELLA domain is responsible for DELLAs stability, which is employed by a GAs receptor Gibberellin insensitive Dwarf 1 (GID1) in GAs dependent and independent manner to lift DELLAs repression to stimulate plant growth under natural environment [13-15].

Rice, barley, tomato, contains only one DELLA gene SLR1 (SLENDER RICE1), SLN1 (SLENDER1), and PROCERA, respectively [9, 16, 17], while LA, CRY in pea [18], $d 8, d 9$ in maize [19] harbor two highly conserved DELLA genes. Additionally, researches on Arabidopsis thaliana revealed the presence of five AtDELLA genes GA-Insensitive (GA), Repressor of ga1-3 (RGA), RGA-Like1 (RGL1), (RGL2), (RGL3). Molecular cloning of the single and multiple loss of the AtDELLA genes in GA deficient mutant ga-1 suggested the overlapping and dissimilar roles of AtDELLAs in regulating GAs stimulated plant growth. For instance, AtGAI and AtRGA have been denoted as notable repressors of the plant vegetative growth $[9,20,21]$, whereas AtRGL 1 and AtRGL2, respectively, are involved in repressing floral augmentation and seed germination [22-25]. AtRGL3 was recently got attention in plant defense by positively regulating the JA and SA mediated response against pathogen infections $[10,26]$

Work over the last decade, the impact of DELLAs on seeded plants productivity has been progressively investigated. Apart from the exogenous splattering of the GAs to increase plant growth by repressing the repressor, one key factor was to alter GAs synthesis by fine-tuning the DELLAs activity for the amelioration of semi-dwarf varieties $[17,27,28]$. This results in enhanced plant tolerance to abiotic stresses, which ultimately increases crops harvest index and survival [29-31]. In addition to this, a recent study identifies a DELLA loss of function semi-dwarf mutant $d s-3$ in oilseed rape, which confers a similar phenotype to previously reported semi-dwarf varieties, with resistance to lodging stress [32]. However, the molecular mechanism and characterization of the $D E L L A$ gene family in B. napus have not been well reported. Moreover, diversification of BnDELLAs during B. napus polyploidization would be of interest. In this study, 10 members of the DELLA protein were systematically characterized and analyzed by their phylogenetic and syntenic relationship, subcellular localization, protein motifs, gene structure, and cis-elements in the B. napus genome. Furthermore, expression profiles of the BnDELLAs in eight different tissues, Root, Mature-Silique, Leaf, Flower, Flower-Bud, Stem, Tips, Seed, were analyzed using the qRT-PCR. BnDELLA genes expression under varied stress conditions, including cold, heat, drought, $\mathrm{ABA}, \mathrm{NaCl}$, and Sclerotinia sclerotiorum were also predicted by publicly available RNA-Seq data. In addition, Gene Ontology (GO) and miRNAs targeting BnDELLA genes were evaluated to designate BnDELLAs roles. These results can provide useful insights to illustrate the multiple functions of the DELLA proteins in $B$. napus and provide a basis for further genetic manipulation toward developing $B$. napus variant with increased stress tolerance to environmental fluctuation.

\section{Results}




\section{Identification and characterization of BnDELLAs}

We have identified 10 BnDELLA genes in B. napus using the known 5 A. thaliana DELLAs (GAl, RGA, RGL 1, RGL2, RGL3) peptide sequences as queries and performed BLASTP search in the $B$. napus genome database (GENOSCOPE http://www.genos cope.cns.fr/brass icanapus/) [33]. To further confirm the BnDELLA proteins integrity in the $B$. napus, we analyze the retrieved sequences in different $B$. napus cultivar genome browser (BnPIR,

http://cbi.hzau.edu.cn/bnapus) and manually corrected the redundant sequence information of the BnDELLAs and named them according to their loci. Based on these methods, we found that each member in 5 AtDELLAs corresponds to multiple homologs in $B$. napus (Table 1). At the same time, 5 DELLA genes in $B$. rapa, 4 in $B$. oleracea, 9 in $B$. juncea, and 5 in $B$. nigra were identified by using the same methods. Furthermore, we found that $10 B n D E L L A s$ members are derived from its progenitor $B$. rapa and $B$. oleracea. The chemical properties of the DELLA proteins in $B$. napus were identified by using the ExPASy (Table 1). The genomic sequence length of the BnDELLAs ranged from the 1524-1740bp, with a molecular weight varying from $55827.07 \mathrm{D}$ to $63323.35 \mathrm{D}$. Moreover, the pl values of the BnDELLA proteins varied from 4.69 to 5.94, which shows that these proteins are highly acidic. Besides, all BnDELLA proteins showed a negative value of the grand average of hydrophobicity (GRAVY), indicating that BnDELLAs are hydrophilic. Subcellular localization signals of the 10 BnDELLA proteins were detected in the nucleus. The names of the BnDELLA genes and their locus id are also shown in (Table1).

\section{Evolutionary relationship and gene structure analysis of BnDELLAs}

The DELLAs evolutionary history among six Brassicaceae species $A$. thaliana, $B$. napus, $B$. rapa, $B$. oleracea, $B$. juncea and $B$. nigra were deduce using the neighbor-joining method. Based on the phylogenetic analysis, 38 DELLA genes in which 5 AtDELLAs, 10 BnDELLAs, 5 BrDELLAs, 4 BoDELLAs, 9 BjDELLAs, and 5 BniDELLAs were cluster into three groups according to the topologies and bootstrap support (Figure 1). Group I contain GAI and RGA clade, Group II holds RGL1 clade, Group III includes RGL2 and RGL3 clade. B. napusDELLA genes were relatively closer to the $A$. thaliana. However, $B$. napus and $B$. rapaDELLAs shows $100 \%$ similarity between each other. In addition, a homolog of AtRGL 1 was not identified in the B. oleracea compared to those of B.napus, B.rapa, $B$. juncea, and $B$. nigra, which might be due to gene loss during the evolution process or maybe the emerging of genome gaps in $B$. oleracea. However, in $B$. napus, Group I, II, III had 4, 2, 4 DELLA members, respectively. DELLA genes grouped into the same subfamily are previously known to have distinct or overlapping functions $[12,22,26,34]$. To recognize the $D E L L A$ genes family diversification in $B$. napus, we have implemented the GSDS web analysis by comparing the CDS and corresponding genomic sequences of AtDELLAs, BnDELLAs, BrDELLAs, BoDELLAs and BjDELLAs. As shown in (Figure 2), members of the $D E L L A$ genes among denoted species are highly conserved and intron-less with only one exon, which indicates $B n D E L L A s$ are the retrogenes. Moreover, the exon location of DELLAs among different phylogenetic related species is conserved, suggesting a similar evolutionary relationship. However, the length of exon among DELLA subfamily was different. For example, BnRGL 1 exon length was smaller than other BnDELLAs members in Group I and Group III, indicating gene structure diversification. In summary, the gene structure of the DELLA genes from different Brassicaceae species are highly conserved with some difference in the exon length (Figure 2).

\section{Multiple sequence alignment and analysis of BnDELLAs motifs}

The putative sequences of the DELLA proteins from B. napus, $A$. thaliana, B. oleracea, $B$. rapa , $B$. juncea, and $B$. nigra were aligned to explore amino acid conservation in $B$. napus. Based on the alignment, we found five homologues DELLA proteins from $A$. thaliana show higher percent amino acid similarity with B. napus (Table S1). Similar to $A$. thaliana, the $B$. napus and other denoted species contain highly conserved DELLA and GRAS domain at the N-terminal and C-terminal region, respectively. The N-terminal DELLA domain is shown to be involved in stabilizing the DELLA genes activity [35, 36], while GRAS domain acts as coregulators to interact with several transcriptional factors and regulators [28, 37, 38] (Figure S1). Moreover, the BnDELLAs amino acid sequence's alignment showed a highly conserved consensus sequence at the $\mathrm{C}$-terminal region, which possibly suggests an evolutionary relationship between $B n D E L L A$ genes (Figure S1). In addition, the presence of the (VHIID-PFYRE-SAW) and two leucine heptad repeats LHRI and LHRII on the C-terminal of the GRAS domain are responsible for the protein interaction $[39,40]$. However, some studies have also proposed DELLA domain lower-affinity with intrinsically unstructured proteins [37, 41]. Overall, the domain arrangements of the DELLA members in B. napus are equivalent to other species. In addition, the secondary structure feature (alpha-helix and Beta sheets) from the AtRGL 1 accessions number (At1G66350.1) was displayed in (Figure S1). However, the predicted secondary structures of all DELLA genes from the denoted plant species were relatively different.

Furthermore, to gain more insights into the putative roles of BnDELLAs diversity in $B$. napus, we generated a graph showing domains and their position on AtDELLAs and BnDELLA protein members. We found that the DELLA and GRAS domains are conserved in all DELLA proteins of $A$ thaliana and $B$.napus, but motifs were unevenly distributed (Figure 3). Every BnDELLA member contains four to sixteen conserved motifs, and their length ranged from six to fifty amino acids. Motif 1 to 13 were identified in all Groups except AtRGL3, BnA1ORGL3, and BnCO9RGL3 lacking motif 12. In which motif 7 and 8 annotated as DELLA domain (Figure S2). Moreover, Motif 14 and 15 were not detected in AtRGL2, BnA05RGL2, BnA05RGL2-2, and Group II, respectively. Motif 16 was detected in AtRGL3, BnA10RGL3, BnCO9RGL3, and in BnA09GAl, BnCO9GAl. Furthermore, Motif 17 was only present in the N-terminal of the AtRGA, BnC07RGA, BnA06RGA, and in BnA05RGL2, BnC05RGL2 genes. Motif 18 was detected in AtRGA and BnA09GAl, BnC09GAl, BnC07RGA, BnA06RGA. In contrast, Group I and AtRGL2, BnA05RGL2, and BnC05RGL2 had an extra motif 19 and 20, respectively. A schematic diagram of all BnDELLAs motifs logos was shown in (Figure S2). These results exhibit that the BnDELLAs subfamilies differ in motif arrangements, indicating the functional divergence of $B n D E L L A$ genes during duplication events. However, proteins with similar motifs arrangements specified the functional similarities among BnDELLA members.

\section{Chromosome location and collinearity analysis}

Chromosomal mapping determined that 10 BnDELLAs distributed on 8 B. napus scaffolds, which has not yet been assembled into a chromosome. Furthermore, no distribution of BnDELLA genes were observed in the scaffoldA01, scaffoldA03, scaffoldA04, scaffoldA07, scaffoldA08, scaffoldC01,

Page $3 / 20$ 
scaffoldC03, scaffoldC04, scaffoldC05, scaffoldC06, and scaffoldC08 (Figure 4). However, six BnDELLA genes, including, BnA02RGL 1, BnA05RGL2, BnA05RGL2-2, BnA06RGA, BnA09GAl, and BnA10RGL3 located on the AA subgenome. While four BnDELLAs, including BnCO2RGL1, BnCO9GAl, BnC07RGA, and BnCO7RGL3 located on the CC subgenome. In contrast, BnA05RGL2 and BnA05RGL2-2 have formed a cluster in the same scaffoldA05, which might result from tandem duplication (Figure 4). These results indicate that BnDELLA genes are unevenly distributed in the $B$. napus genome.

Furthermore, by using the BLAST and MCScanX methods, we investigate the 6 segmental duplication pairs such as, BnA06RGA/BnC09GAl, BnC07RGA/BnA09GAl, BnA02RGL 1/BnC09RGL3, and one tandem duplication BnA05RGL2/BnA05RGL2-2 were determined (Figure 5), which exhibits that during evolution segmental duplication events were the main reason for the divergence of $D E L L A$ genes in $B$. napus. In addition, comparative synteny of $D E L L A$ gene pairs between $B$. napus and $A$. thaliana was conducted (Figure 6). The results show that 10 BnDELLA genes of $B$. napus are associated with AtDELLAs, which are more than one ortholog's copies in BnDELLAs. For instance, AtGAland AtRGA show synteny relationships with BnA09GAl, BnCO9GAl, BnA06RGA and BnC07RGA, suggesting that AtDELLAs genes might contribute to the evolution of the BnDELLAs family. Moreover, we evaluate the pressure of selective constrains on each pair of duplicated BnDELLA genes, and calculated the nonsynonymous (Ka) and synonymous (Ks) ratio (Table S2, Figure S3). Our findings showed that all of the BnDELLA pairs had the Ka/Ks ratio lower than 1, implying that BnDELLA genes experienced robust purifying selective pressure.

\section{Prediction of the bn-microRNAs putative targets sites of BnDELLAs}

The regulatory purpose of DELLAs and their interacting proteins have been characterized widely in various plant species; however, a possible underlying posttranscriptional modification of DELLAs in response to environmental stresses is still unclear [42-44]. It has been reported that miRNAs play a significant role in transcriptional and post-transcriptional level to modulate gene expression under stresses $[45,46]$. To identify miRNAs interaction with $B n D E L L A s$ isoforms, we obtained the bn-miRNAs data from $B$. napus comprehensive study to predict the targeted BnDELLA genes sites. We found that $10 B n D E L L A$ genes from $B$. napus were unambiguously complementary to the 18 known $B$. napus miRNAs. These miRNAs length reached from 1-24 base pairs, with 11 nt being the most frequent in all BnDELLAs (Table 2). Target prediction shows that BnDELLAsBnaC07RGA and BnaA09GA/are targeted by 2 well-known miRNAs, bna-miR6029 and bna-miR6031, respectively. Among the other bn-miRNAs identified in our analysis, bna-miR2111a, bna-miR166a are found to be involved in targeting the BnRGL 1. In contrast, bna-miR172b targets BnA02RGL 1 and BnA05RGL2. Additionally, bna-miR390a and bna-miR168a are found to target BnRGL3. Based on this analysis, we perceived that $B$. napus miRNA strongly targets $B$. napus both $A$ and $C$ genome to regulate BnDELLAs gene expression under constant stress to stabilize plant growth and defense tradeoff.

\section{cis-element analysis in promoter regions of BnDELLAs and their distribution}

Physiological and molecular studies on DELLAs suggested their role in multiple hormonal signaling pathways by interacting with a wide variety of transcriptional regulators and transcriptional factors. However, the molecular mechanism of interaction and regulation of $D E L L A$ genes are quite unclear. To gain more insights into the potential function and regulatory mechanism of the BnDELLAs, we analyzed the cis-regulatory elements in the $1500 \mathrm{bp}$ upstream promoter region of the BnDELLAs by using the Plant-CARE database and divided them into four categories (Figure 7A). We found that the individual DELLA gene in $B$. napus contains multiple cis-acting elements (Table S3), nearly all of the BnDELLA genes promoters have CAAT-box, TATA-box, light, stress, hormone, and development related responsive cis-elements. However, the distribution and numbers differed significantly between the $B \cap D E L L A$ genes (Figure 7B). In detail, BnA09GAl, BnA02RGL 1 has a higher number of light and hormone-responsive elements. In contrast, BnA06RGA, BnA05RGL2, and BnA10RGL3 carried a higher number of stress and development related cis-elements, respectively. However, some of the cis core elements were only found in some $B n D E L L A s$. For example, GC-motif (enhancer-like element involved in anoxic specific inducibility), DRE-core (cis-acting regulatory element regulate cold stress induce dehydration), and 3-AF binding site (part of a conserved DNA module array CMA3) were found in BnA06RGA and BnCO7RGABnDELLA members. Similarly, GATA-motif (cis-acting regulatory element involved in light-responsive floral, hypocotyl, and seed development), AT-Rich sequence (cis-element for maximal elicitor-mediated activation) were present in BnCO9RGA and BnA10RGA. ATCT-motif (Part of a conserved DNA module involved in light responsiveness), GapBox (cis-acting element related to light-responsive GapA gene) were present in BnC02RGL 1 and BnA02RGL 1, respectively. Moreover, AuxRR-Core (cis-acting regulatory element involved in auxin responsiveness) was only found in BnA05RGL2 and BnA05RGL2-2. In contrast, 02-site (cis-regulatory element involved in zein metabolism regulation) was absent in all BnDELLAs except BnCO9RGL3 and BnA10RGL3 (Figure 7A). These results showed that BnDELLA genes contain a wide variety of stress and defense-related cis-elements compared to development, light, and hormone-responsive cis-elements, suggesting the $B n D E L L A s$ diverse function in response to various biotic and abiotic conditions.

\section{Transcriptomic and qRT-PCR analysis of BnDELLAs in different tissues}

The candidate BnDELLA genes expression transcriptomic data from the different tissues of the B. napus variety "Zhongshuang 11" (ZS11) were obtained from the BnTIR database http://yanglab.hzau.edu.cn/BnTIR. The extracted data normalized by log2 fold change and heatmap were generated. As shown in (Figure 8), the expression pattern of the 10 BnDELLA genes was different among various tissues, which points out that the additional copies of the homologs $B n D E L L A$ genes show variations in expression during plant development. This can provide important insight into these genes' distinct roles in $B$. napus. To better understand the expression pattern of the BnDELLA genes, we performed qRT-PCR in 8 primary tissues (Root, Mature-Silique, Leaf, Flower, Flower-Bud, Stem, Tips, Seed) of $B$. napus variant ZS11. We found a strong correlation between the transcriptomic and our qRT-PCR results (Figure 9). On the whole, BnGAI and $B n R G A$ are highly expressed in the stem and tips, while BnRGL 1 and $B n R G L 2$ were mainly expressed in the floral organs and seed, respectively. Conversely, in our qRT-PCR analysis, BnRGL3 show minimal expression in any tissues. However, based on the transcriptomic data analysis, $B n R G L 3$ expression was highly observed in the silique. The contradiction between the qRT-PCR and transcriptomic data, especially in the BnRGL3 expression, might be due to the harvesting of silique at 6 and 28 days after flowering, which show the complex variation of the BnDELLA genes expression at various stages of

Page $4 / 20$ 
plant development. These results indicate that the expression of BnDELLA genes at multiple plant tissues might play an indispensable role in regulating gibberellins and other phytohormones signals to mediate plant growth and survival tradeoff under constant stress conditions.

\section{Expression analysis of BnDELLAs under different stress}

To further explore and gain more insights into possible BnDELLAs function. We used the publicly available RNA-seq data to analyzed the BnDELLA genes expression under different stress conditions, such as MA, CA, FA, DT, HT, ABA, NaCl, and Sclerotinia sclerotiorum. Our predicted RNA-seq data show that the $B n D E L L A s$ gene expression significantly changed upon different stress treatments. For instance, BnRGL2 was up-regulated by all denoted stresses except in drought and salt (Figure 10). Whereas, BnGA/ show induced expression in response to MA, HT, DT, and salinity. In contrast, BnA10RGL3, BnCO9RGL3 almost exhibits reduced expression in response to heat, drought, ABA, and salt treatment. However, induced expression was observed during cold and Sclerotinia sclerotiorum treatment. Many previous studies on AtDELLA genes had provided evidence of their distinct and fundamental role in regulating plant physiology under abiotic and biotic stress [24, 47-49], suggesting the importance of BnDELLA genes in improving stress tolerance.

\section{Gene Ontology}

In order to understand the functional regulatory mechanism, we used the AtDELLA orthologous pairs of the A. thaliana to performed GO enrichment analysis. Three common categories of GO terms were observed including, biological process (BP), cellular component (CC), and molecular function (MF). In the MF category, DELLA genes are highly enriched in binding (G0:0003700), (G0:0005515), and transcriptional regulation activity (G0:0140110). CC is enriched in the nucleus (GO:0005634), which exhibits that DELLAs are nuclear proteins. Similarly, most of the GO terms (G0:0009737, G0:0009739, G0:0009740, GO:0009753, GO:0042538, GO:0009863, G0:0072593, G0:0009651, G0:0009908, GO:2000033, G0:0030154, G0:0010187, G0:0009938, G0:0006355, G0:0010218, G0:0009723) were abundant in biological process, which show response to hormones and stresses (Figure 11, Table S7). This G0 enrichment results suggested that the BnDELLAs gene plays a crucial role in regulating hormonal signaling in response to stresses, which is consistent with previous studies $[9,50-52]$.

\section{Discussion}

In recent years, the cultivation of the $B$. napus declining due to the versatility of the environment. DELLAs are well known as the master negative coregulators to mediate cross-talk between GAs and multiple hormonal signals to modulate plant growth and survival tradeoff, responding to abiotic and biotic conditions $[8,10]$. Previous reports on seeded plants had identified 1, 2, and 5 DELLA genes in Oryza sativa [16], Pisum sativum [18], and A. thaliana, respectively. Cloning and modulation of DELLA proteins in these plants resulted in increased harvest index, seed quality, tillering, flower timing, and stress tolerance. For example, overaccumulation of the DELLA protein enhances the submergence tolerance [53], salt stress [54], and shade avoidance [55, 56], which significantly improve plant fitness. In contrast, reduced DELLA protein expression reduces tillering $[57,58]$ and seed dormancy [59], suggesting increase seed weight and germination. However, despite the impressive insights gained from these studies, no previous comprehensive analysis of $D E L L A$ genes had carried out in $B$. napus. In this study, a total of 10 BnDELLA genes have been identified in $B$. napus. We analyzed phylogenetic relation, gene structure, motif composition, physicochemical properties, gene duplication, miRNA prediction, and cis-element analysis in the promoters. Moreover, qRT-PCR and published RNA-seq data were analyzed to disclose the expression profiling of the BnDELLA genes' and their roles under different stress conditions.

\section{Characterization, and evolution of the B. BnDELLAs in $B$. napus}

B. napus is an allotetraploid (AACC) crop that originated from the hybridization of two diploid progenitors $B$. rapa (AA) and $B$. oleracea (CC) [60]. In our study, we identified 10 full-length putative sequences of BnDELLA genes in $B$. napus, which are approximately equal to 5 in $B$. rapa, and 4 in $B$. oleracea. According to phylogenetic analysis, BnDELLA genes are classified into three groups (Figure 1), Group I contain BnGA/and BnRGA; Group II contains BnRGL 1; Group III contains BnRGL2, and BnRGL3, which are based upon the identified AtDELLAs (Figure1). This suggests the possibility of similar evolutionary trajectories and genome duplication. As a result, one AtDELLA was duplicated into two homologs genes in B. napus (Figure 6). Additionally, we also find that the expansion of $B n D E L L A$ genes in $B$. napus is drive by the tandem and segmental duplication during the evolutionary process (Figure 5 ). However, the number of tandem duplications is much lower than the segmental duplication, suggesting that segmental duplication plays a higher role in expanding $B n D E L L A$ genes. Additionally, the calculated $\mathrm{Ka} / \mathrm{Ks}$ ratios of BnDELLA gene pairs were less than < 1 (Table S2), suggesting BnDELLAs are under strong purifying selection during the duplication process.

Furthermore, we also identify that coding regions of the $A$. thaliana, $B$. napus, $B$. rapa, $B$. oleracea, and $B$. juncea DELLA genes contain a single exon and does not have any introns (Figure 2), indicating the lower expression of DELLA genes in plants. However, previous studies indicated that genes with compact structures maybe expressed rapidly in response to stresses [61, 62]. For instance, the intronless gene BnRGL3 show reduced expression in most tissues (Figure 9), but expressed rapidly in response to Sclerotinia sclerotiorum infection (Figure 10, Table S6). Moreover, exon composition exhibited the higher evolutionary conservation of DELLA genes among Brassicaceae species. In addition, chromosomal mapping indicated that 5 and $4 B n D E L L A$ genes are located on the proximal or the distal ends of AA and CC subgenome, respectively (Figure 4), which exhibits homologs of BnDELLAs might play a similar role in biological function as AtDELLAs. Significant progress has been made to characterize the distinct roles of the DELLA gene family in Arabidopsis and other plant species; however, there is little progress in understanding the role of BnDELLAs in $B$. napus. Our comparative analysis of the sequence diversification suggests that BnDELLA genes contain two highly conserved N-terminal DELLA and C-terminal GRAS domain (Figure S1). The N-terminal DELLA domain is involved in the DELLA genes' stability [63]. In contrast, the C-terminal GRAS domain interacts with a wide variety of transcriptional factors and transcriptional regulators in response to stresses $[39,40]$. DELLA genes are localized in the nucleus, suggesting their transportation regulation role [39]. In addition, motif numbers and 
their composition between BnDELLA genes are unevenly distributed, indicating the domain shuffling in the protein structure of the BnDELLA genes (Figure 3). However, we observe that motif 1-11 and 13 are common in all BnDELLA genes.

A recent study has hypothesized that the OsmiR396 putatively regulated by the rice DELLA gene $S L R 1$, targeting GA-responsive growth-regulating factors (GRFs) to inhibit growth promotion in rice [64]. In this study, a total of 18 bn-miRNAs were predicted to putatively regulate all BnDELLA genes (Table 2). In which, BnC07RGA and BnA09GA/ are potentially regulated by the two-known miRNAs bna-miR6029 and bna-miR6031, respectively. In compliance with this, a recent study has shown that the increased expression of the bna-miR6029 regulates fatty acid biosynthesis to mediate seed development [65]. Thus, we speculate that the BnDELLA genes were the most likely targeted genes by the predicted bn-miRNAs to regulate plant growth and survival tradeoff under constant exogenous or endogenous stimuli. However, further investigation is needed to elucidate the miRNA process with $B n D E L L A$ genes.

\section{Gene Expression profiling disclose the potential role of the BnDELLA genes}

DELLA proteins are previously well described as master repressors of GAs signaling to modulate plant physiology [66, 67]. In recent studies, they have been identified for interacting with various transcriptional factors and transcriptional regulators to regulate crosstalk between GAs and multiple phytohormones in favor of plant survival under constant stress conditions $[9,68,69]$. However, the effect of DELLA proteins on growth and productivity is much larger than is widely known. Researches on A. thaliana have identified five AtDELLA genes GA-Insensitive (GAI), Repressor of ga1-3 (RGA), RGA-Like1 (RGL 1), (RGL2), (RGL3). Cloning and sequencing of these AtDELLA genes reported the distinct and overlapping role in regulating GAs stimulated plant growth. For instance, AtGA/ and AtRGA control hypocotyl cell division and floral induction $[20,21,70]$. AtRGL 1 and AtRGL2 are involved in modulating leaf senescence, male sterility, and seed germination [23, 24]. While AtRGL3 has been reported to contribute plant defense in response to biotic stresses [10, 26, 71]. Consistent with this, our qRT-PCR and predicted RNA-Seq data (Table S4.1) indicated that the individual BnDELLA gene subfamily exhibits significant varied expression and response to biotic and abiotic stress. For instance, BnRGL2 was up-regulated by all tested stresses except in drought, salinity, and Sclerotiniasclerotiorum (Figure 10). Whereas, $B n G A /$ is highly expressed in stems and shows a response to MA, HT, DT, and salinity. In contrast, BnRGL3 almost exhibits reduced expression in response to heat, drought, and $\mathrm{ABA}$ treatment. However, induced expression was observed during cold and $\mathrm{NaCl}$ treatment. These findings are consistent with studies that have also been found on their homologs in $A$. thaliana [72, 73]. Moreover, previous studies also confirmed the increased expression of the AtRGL3 in response to the biotic stresses in $A$. thaliana [10, 26, 71]. As expected, increased expression of the BnA09RGL3 and BnC09RGL3 was observed after 24 hours of Sclerotinia sclerotiorum infection (Figure 10), suggesting the BnRGL3 vital role in mediating $B$. napus survival under constant stress condition.

Furthermore, BnRGL2 homolog in A. thalianaAtRGL2 is denoted as an essential component to positively regulate ABA responses to promote seed dormancy [74-76]. In our qRT-PCR and RNA-seq analysis, we found that BnRGL2 was mainly expressed in the seeds and showed induced expression after 4 hours of ABA treatment, but eventually reduced after 24 hours of $A B A$ treatment. However, further experimental studies are required to gain more insights into the $B n D E L L A s$ in the ABA signal transduction pathway. In addition, during salt stress, the transcripts of the BnA09GAl, BnCO9GAl, and BnCO9RGL3 were up-regulated, whereas the rest of the BnDELLA genes were down-regulated (Figure 10), suggesting the importance of $B n G A /$ in susceptibility to severe salt stress. Importantly the link of $A t G A$ / with salt stress has been identified, which confirmed the enhanced salt tolerance by restraining the plant growth [77, 78]. These results indicate the functional diversification of the BnDELLA genes under varying environmental conditions.

Moreover, in our study, we found diverse cis-elements in BnDELLAs promoter, including light-responsive, hormones responsive, and stress-related elements (Figure 7), but their distribution is uneven. For instance, BnA02RGL 1, BnC02RGL 1, and BnA09RGL3, BnC09RGL3 had 2 ABREs in their promoter regions, while $B n A 05 R G L 2$ and BnA05RGL2-2 had no ABREs, although they were considered to induced ABA response differently. Additionally, BnA05RGL2 and BnA05RGL22 had 1 MBS cis-element in their promoter regions. Intriguingly, the BnRGL2 gene relative expression was not observed in the drought treatment (Figure 10). Thus, these findings indicate the presence of unidentified cis-elements and signify that the expression of BnDELLAs might be regulated through posttranscriptional modification [42,44], which determines the antagonistic and synergistic effects of BnDELLAs in response to environmental fluctuations. To further investigate the biological process associated with BnDELLA genes, we had performed the GO enrichment analysis. As shown in (Figure 11), BnDELLAs involved in protein and DNA-binding and were located in the nucleus. Additionally, the majority of the BnDELLAs participated in hormonal regulation in response to biotic and abiotic stresses. To better understand the various roles of the BnDELLAs genes under biotic and abiotic stress conditions, further experimental studies are needed, which will help consolidate our understanding of plant ontogenesis and facilitate the enhancement of agronomic techniques to improve $B$. napus yield.

\section{Conclusions}

In our study, we identified and characterized the BnDELLA genes in B. napus. A total of $10 B n D E L L A$, genes are identified in $B$. napus genome, which were classified into three groups. Group I carry BnGAl, and BnRGA. Group II contains BnRGL1. BnRGL2, BnRGL3 belong to Group III. All of the BnDELLA genes are closely related to the Arabidopsis thaliana five $D E L L A$ genes, suggesting a comparable function and structure. The motifs composition in all BnDELLA genes is uneven; however, every BnDELLA gene contains 12 highly conserved motifs, encoding DELLA and GRAS domain. In addition, no introns insertion patterns were predicted in the DELLA and GRAS domains of the BnDELLA genes. Furthermore, phylogenetic and synteny study of the DELLA genes between $B$. napus and $A$. thaliana provides useful hints or evolutionary features of the BnDELLA genes. Moreover, cis-element analysis and miRNAs targets were also predicted. Quantitative reverse-transcription polymerase chain reaction (qRT-PCR) analysis has exhibited the BnDELLA genes' diverse expression patterns in various plant development stages. Furthermore, induced expression of the BnDELLA genes by different stress treatments were also determined by RNA-seq analysis. Overall, these results proved useful clues into the evolutionary relationship and potential functions of the BnDELLAs, which will be useful for further genetic manipulation toward developing $B$. napus variant with increased stress tolerance to environmental fluctuation.

\section{Methods}




\section{Identification and protein sequence analysis of BnDELLAs}

In order to search DELLA gene family in B. napus, peptide sequence of the 5 DELLA genes from A. thaliana genome database (http://www.arabidopsis.org/) with corresponding Gene ID (At1G14920.1, At2G01570.1, At1G66350.1, At3G03450.1, At5G17490.1) were retrieved and used as queries to perform BLAST P search in $B$. napus Genome browser (BnPIR, http://cbi.hzau.edu.cn/bnapus), and (GENOSCOPE, http://www.genoscope.cns.fr/brass icana pus). Those from $B$. oleracea, B. rapa, B. juncea, and B. nigra were downloaded from Brassica Database (BRAD, http://brassicadb.org/brad). The sequences with $80 \%$ similarity were selected, and incorrectly or repeated sequences were manually re-annotated for DELLA domain analysis in the scan ScanProsite (http://www.expasy.ch/ tools/scanprosite/) and InterProScan (http://www.ebi.ac.uk/ Tools/InterProScan/). The protein sequences were then used to calculate the isoelectric point (pl), molecular weight (MW) and number of amino acids by the ProtParm tool (http://web.expasy.org/). Furthermore, prediction of subcellular location pattern of each BnDELLA was carried out using the web-server Plant-mPLoc (http://www.csbio.sjtu.edu.cn/bioinf/plant-multi/) [79], and ProtComp v.9.0 in softberry (http://linux1.softberry.com/).

\section{Phylogentic and gene structure assessment of the DELLA in B. napus, $A$. thaliana, B. rapa, B. oleracea, B, juncea, B. nigra}

Putative peptide sequences from the six Brassicaceae species B. napus, $A$. thaliana, $B$. rapa, B. oleracea, $B$. juncea, and $B$. nigra aligned using the MUSCLE (https://www.ebi.ac.uk/Tools/msa/muscle) with default parameters. Aligned sequences were then used to construct the evolutionary tree with the MEGA 10.2 software by the neighbor-joining (NJ) method [80]. The authenticity of the tree was tested by performing 1000 bootstrap replications. The Newick format of the phylogenetic tree was then uploaded to iTOL web server (http://itol.embl.de/) for better visualization. Furthermore, genomic and coding sequences of the B. napus, B.oleracea, B.rapa, B. juncea and A. thaliana DELLA genes were rendered in Gene Structure Display Server (GSDS2.0) (http://gsds.cbi.pku.edu.cn) to predict gene structure and exon/intron location.

\section{Sequence alignment and evaluation of BnDELLAs motifs}

To classify the DELLAs characteristic domains in the $B$. napus, we have aligned the 38 DELLAs codding sequence from $B$. napus, $A$. thaliana, $B$. rapa, $B$. oleracea, $B$. juncea, and $B$. nigra by using the Muscle option in the MEGA 10 with default parameters. Furthermore, Motif Elicitation version 5.1 .1 (MEME http://meme-suite.org/tools/meme) was used to identify the conserved motifs in the BnDELLAs with the maximum motif search set to 20 , and other parameters are set to default. Any repetitions were considered motifs sites that spread throughout the sequence [81]. Further annotation of the identified motifs was implemented by the InterProScan (InterPro ebi.ac.uk). The conserved motifs were visualized by using the TBtools software [82]. Additionally, the secondary structure of the BnDELLA proteins is carried by PSIPRED (http://bioinf.cs.ucl.ac.uk/PSIPRED).

\section{Chromosome location, collinearity analysis, and site-specific selection assessment and testing}

BnDELLAs detailed chromosome location was acquired from the GFF genome file downloaded from Brassica napus genomic database (BnPIR, http://cbi.hzau.edu.cn/bnapus), and mapped the predicted location on the chromosome by using the TBtools software with colored gene names indicated as relative position. Gene duplication events were identified by aligning the BnDELLA genes sequences using BLASTP, and MCScanX was used to characterize the BnDELLAs into a tandem and segmental duplication [83]. Furthermore, the synteny map of DELLAs orthologous among $B$. napus and $A$. thaliana were obtained by the custom phyton script. For examining the site-specific selection, a Bayesian inference approach Selecton Server (http://selecton.tau.ac.il/ [84] was used to predict the positive and purifying selection. Besides this, we also calculated the synonymous $(K s)$ and Non-synonymous mutation (Ka) at each codon by KaKs_Calculator 2.0 [85]. In addition, BnDELLA gene pairs' divergence time was presumed using the formula $\mathrm{T}=\mathrm{Ks} / 2 \mathrm{r}$ with $\mathrm{r}\left(1.5 \times 10^{-8}\right)$ representing neutral substitution per site per year [86].

\section{miRNA target prediction and cis-acting elements regulatory analysis}

To predict the miRNAs regulating BnDELLA genes. We obtained the $B$. napus stem-loop and mature miRNA sequences from the PNRD (http://structuralbiology.cau.edu.cn/PNRD/index.php) [87] and miRbase (http://www.mirbase.org/) database. Then we submitted bn-miRNAs identified sequences and Genomic segment sequences of the BnDELLA genes into psiRNATarget server http://plantgrn.noble.org/psRNATarget with default parameters. For cis-element analysis, $1500 \mathrm{~kb}$ upstream promoter sequence from the translation start site of the BnDELLAs were scrutinized in the plant care database (http://bioinformatics.psb.ugent.be/webtools/plantcare/html/) [88], and distribution of the cis-acting elements visualized by TBtools software [82].

\section{RNA extraction and quantitative Real-time PCR analysis of BnDELLAs in different tissues}

To explore the expression pattern of the BnDELLA genes, we extracted the RNA of B. napus variety ZS11 different tissues (Roots, Mature-silique, Leaf, Flower, Flower-Bud, Stem, Tips, Seed) using Trizol (Invitrogen, Carlsbad, CA). Total RNA was then employed to produce cDNA with HiScript III-RT SuperMix for qPCR (Vazyme, China) according to the manufacturer's instructions. Real-time fluorescence quantitative analysis (qRT-PCR) was performed by Thermo Fisher Scientific QuantStudio 5 Real-Time PCR system with three independent replicates, and Actin was used as an internal control. The $2^{-\Delta \Delta} \mathrm{Ct}$ method was implemented to measure the relative gene expression level of BnDELLA genes, and the results were visualized using GraphPad Prism8.0 software [89]. All of

Page $7 / 20$ 
the gene-specific primers used in this study were designed by the Beacon primer design program (Primer Biosoft International, Palo Alto, CA) and listed in (Table S5).

\section{Gene ontology and expression pattern analysis of BnDELLAs under abiotic biotic stress}

The BnDELLA genes functional properties were analyzed using the online web server DAVID (https://david.ncifcrf.gov/) and panther (http://go.pantherdb.org/webservices/go/overrep.jsp) to conduct Gene Ontology enrichment analysis. The predicted GO terms were annotated using the TBtools software. In addition, expression profiles of BnDELLA genes under heat, drought, cold, ABA induce, salt and Sclerotinia sclerotiorum stress condition were obtained from the publicly available transcriptomic data sets (GSE156029, GSE129220) [90], (CRA001775) [91], and (GSE81545) [92]. The differential expression analysis was performed using DSEeq2 package in R. The predicted values were normalized by log2 fold change, and heatmap was generated via TBtools.

\section{Abbreviations}

B. napus: Brassica napus; A. thaliana: Arabidopsis thaliana; B. rapa: Brassica rapa; B. oleracea: Brassica oleracea; $B$. nigra: Brassica nigra; $B$. juncea: Brassica juncea: AtDELLA: Arabidopsis thaliana DELLA; BnDELLA: Brassica napus DELLA; GAs: Gibberellins; Go: Gene ontology; BLASTP: Basic local alignment search tool-protein; MW: Molecular weight; MEME: Motif elicitation; bn-miRNAs: Brassica napus microRNA; ZS11: Zhongshuang 11; GID1: Gibberellin insensitive Dwarf 1; GRAVY: Grand average of hydrophobicity; pl: Isoelectric point; ABA: Abscisic acid; NaCl: Sodium chloride.

\section{Declarations}

\section{Author Contributions}

RS and ZKM designed the experiment. RS, TJ, PD and YG carried out the data analysis. RS wrote the manuscript draft and ZKM revised and edit the manuscript. ZKM and RS provided the RNA-seq data. RS, TJ, PD and YG provided the experimental materials. XLT supervised the manuscript. All authors read and approved the final manuscript.

\section{Funding}

This work was supported by the National Key Research and Development Program of China (2016YFD0101904; 2016YFD0100305) and the National Natural Science Foundation of China (31671720), and the Distinguished Scholars Research Foundation of Jiangsu University (No. 10JDG134).

\section{Conflicts of Interest}

The authors declare no conflict of interest.

\section{Availability of the data material}

BnDELLA genes expression transcriptomic data from the different tissues of the B. napus variety "Zhongshuang 11" (ZS11) were obtained from the BnTIR database http://yanglab.hzau.edu.cn/BnTIR. RNA-seq data of B. napus under heat, drought, cold; ABA induce, salt and Sclerotinia sclerotiorum stress condition were obtained from the publicly available transcriptomic data sets GSE156029, GSE129220; CRA001775, and GSE81545, respectively. All additional datasets supporting the results of this article are included within the article and supplementary tables.

\section{References}

1. Kimber D, McGregor D: Brassica oilseeds: production and utilization: Cab international; 1995.

2. Zhang X, Zhang C, Xing L, Wang H: Investigation on 2008'low temperature and freeze injure on winter rape along Yangtze River. Oil Crop Science 2008, 30(1):122-126.

3. Boter M, Calleja-Cabrera J, Carrera-Castano G, Wagner G, Hatzig SV, Snowdon RJ, Legoahec L, Bianchetti G, Bouchereau A, Nesi N et al: An Integrative Approach to Analyze Seed Germination in Brassica napus. Frontiers in Plant Science 2019, 10:1342-1342.

4. Huot B, Yao J, Montgomery BL, He SY: Growth-defense tradeoffs in plants: a balancing act to optimize fitness. Molecular plant 2014, 7(8):1267-1287.

5. Vanstraelen M, Benkova E: Hormonal Interactions in the Regulation of Plant Development. Annu. Rev. Cell Dev. Biol. 2012, 28:463-487.

6. Albrecht T, Argueso CT: Should I fight or should I grow now? The role of cytokinins in plant growth and immunity and in the growth-defence trade-off. Ann Bot 2017, 119(5):725-735.

7. Patel D, Franklin KA: Temperature-regulation of plant architecture. Plant signaling behavior 2009, 4(7):577-579.

8. Hedden P, Thomas SG: Annual plant reviews, the gibberellins, vol. 49: John Wiley \& Sons; 2016.

9. Daviere JM, Achard P: A Pivotal Role of DELLAs in Regulating Multiple Hormone Signals. Molecular Plant 2016, 9(1):10-20. 
10. Li Y, Yang Y, Hu Y, Liu H, He M, Yang Z, Kong F, Liu X, Hou X: DELLA and EDS1 Form a Feedback Regulatory Module to Fine-Tune Plant Growth-Defense Tradeoff in Arabidopsis. Molecular plant 2019, 12(11):1485-1498.

11. Koorneef M, Elgersma A, Hanhart CJ, van Loenen-Martinet EP, van Rijn L, Zeevaart JAD: A gibberellin insensitive mutant of Arabidopsis thaliana. Physiol. Plant. 1985, 65(1):33-39.

12. Peng J, Carol P, Richards DE, King KE, Cowling RJ, Murphy GP, Harberd NP: The Arabidopsis GAl gene defines a signaling pathway that negatively regulates gibberellin responses. Genes Dev. 1997, 11(23):3194-3205.

13. Ueguchi-Tanaka M, Nakajima M, Katoh E, Ohmiya H, Asano K, Saji S, Hongyu X, Ashikari M, Kitano H, Yamaguchi I et al: Molecular interactions of a soluble gibberellin receptor, GID1, with a rice DELLA protein, SLR1, and gibberellin. The Plant Cell 2007, 19(7):2140-2155.

14. Yamamoto Y, Hirai T, Yamamoto E, Kawamura M, Sato T, Kitano H, Matsuoka M, Ueguchi-Tanaka M: A rice gid1 suppressor mutant reveals that gibberellin is not always required for interaction between its receptor, GID1, and DELLA proteins. The Plant Cell 2010, 22(11):3589-3602.

15. Fuentes S, Ljung K, Sorefan K, Alvey E, Harberd NP, Østergaard L: Fruit growth in Arabidopsis occurs via DELLA-dependent and DELLA-independent gibberellin responses. The Plant Cell 2012, 24(10):3982-3996.

16. Ikeda A, Ueguchi-Tanaka M, Sonoda Y, Kitano H, Koshioka M, Futsuhara Y, Matsuoka M, Yamaguchi J: Slender rice, a constitutive gibberellin response mutant, is caused by a null mutation of the SLR1 gene, an ortholog of the height-regulating gene GAI/RGA/RHT/D8. The Plant Cell 2001, 13(5):999-1010.

17. Chandler PM, Marion-Poll A, Ellis M, Gubler F: Mutants at the Slender1 locus of barley cv himalaya. Molecular and physiological characterization. Plant Physiol. 2002, 129(1):181-190.

18. Weston DE, Elliott RC, Lester DR, Rameau C, Reid JB, Murfet IC, Ross JJ: The pea DELLA proteins LA and CRY are important regulators of gibberellin synthesis and root growth. Plant Physiol. 2008, 147(1):199-205.

19. Lawit SJ, Wych HM, Xu DP, Kundu S, Tomes DT: Maize DELLA Proteins dwarf plant8 and dwarf plant9 as Modulators of Plant Development. Plant Cell Physiol. 2010, 51(11):1854-1868.

20. Dill A, Sun TP: Synergistic derepression of gibberellin signaling by removing RGA and GAl function in Arabidopsis thaliana. Genetics 2001, 159(2):777785.

21. King KE, Moritz T, Harberd NP: Gibberellins are not required for normal stem growth in Arabidopsis thaliana in the absence of GAI and RGA. Genetics 2001, 159(2):767-776.

22. Lee SC, Cheng H, King KE, Wang WF, He YW, Hussain A, Lo J, Harberd NP, Peng JR: Gibberellin regulates Arabidopsis seed germination via RGL2, a GAI/RGA-like gene whose expression is up-regulated following imbibition. Genes Dev. 2002, 16(5):646-658.

23. Ravindran P, Verma V, Stamm P, Kumar PP: A Novel RGL2-DOF6 Complex Contributes to Primary Seed Dormancy in Arabidopsis thaliana by Regulating a GATA Transcription Factor. Molecular Plant 2017, 10(10):1307-1320.

24. Chen L, Xiang S, Chen Y, Li D, Yu D: Arabidopsis WRKY45 Interacts with the DELLA Protein RGL1 to Positively Regulate Age-Triggered Leaf Senescence. Molecular Plant 2017, 10(9):1174-1189.

25. Gomez MD, Barro-Trastoy D, Fuster-Almunia C, Tornero P, Alonso JM, Perez-Amador MA: Gibberellin-mediated RGA-LIKE1 degradation regulates embryo sac development in Arabidopsis. Journal of Experimental Botany 2020, 71(22):7059-7072.

26. Wild M, Achard P: The DELLA protein RGL3 positively contributes to jasmonate/ethylene defense responses. Plant Signaling \& Behavior 2014, 8(4):e23891-e23891.

27. Evenson RE, Gollin D: Assessing the impact of the Green Revolution, 1960 to 2000. Science 2003, 300(5620):758-762.

28. Asano K, Hirano K, Ueguchi-Tanaka M, Angeles-Shim RB, Komura T, Satoh H, Kitano H, Matsuoka M, Ashikari M: Isolation and characterization of dominant dwarf mutants, SIr1-d, in rice. Mol. Genet. Genomics 2009, 281(2):223-231.

29. Cheng H, Qin L, Lee S, Fu X, Richards DE, Cao D, Luo D, Harberd NP, Peng J: Gibberellin regulates Arabidopsis floral development via suppression of DELLA protein function. Development 2004, 131(5):1055-1064.

30. Achard P, Gong F, Cheminant S, Alioua M, Hedden P, Genschik P: The cold-inducible CBF1 factor-dependent signaling pathway modulates the accumulation of the growth-repressing DELLA proteins via its effect on gibberellin metabolism. The Plant Cell 2008, 20(8):2117-2129.

31. Felipo-Benavent A, Úrbez C, Blanco-Touriñán N, Serrano-Mislata A, Baumberger N, Achard P, Agustí J, Blázquez MA, Alabadí D: Regulation of xylem fiber differentiation by gibberellins through DELLA-KNAT1 interaction. Development (Cambridge) 2018, 145(23).

32. Zhao B, Li H, Li J, Wang B, Dai C, Wang J, Liu K: Brassica napus DS-3, encoding a DELLA protein, negatively regulates stem elongation through gibberellin signaling pathway. Theor Appl Genet 2017, 130(4):727-741.

33. Chalhoub B, Denoeud F, Liu S, Parkin IA, Tang H, Wang X, Chiquet J, Belcram H, Tong C, Samans B: Early allopolyploid evolution in the post-Neolithic Brassica napus oilseed genome. Science 2014, 345(6199):950-953.

34. Wen CK, Chang C: Arabidopsis RGL1 encodes a negative regulator of gibberellin responses. Plant Cell 2002, 14(1):87-100.

35. Ueguchi-Tanaka M, Ashikari M, Nakajima M, Itoh H, Katoh E, Kobayashi M, Chow TY, Hsing YIC, Kitano H, Yamaguchi I et al: GIBBERELLIN INSENSITIVE DWARF1 encodes a soluble receptor for gibberellin. Nature 2005, 437(7059):693-698.

36. Murase K, Hirano Y, Sun TP, Hakoshima T: Gibberellin-induced DELLA recognition by the gibberellin receptor GID1. Nature 2008, 456(7221):459-463.

37. Hauvermale AL, Ariizumi T, Steber CM: Gibberellin Signaling: A Theme and Variations on DELLA Repression. Plant Physiol. 2012, 160(1):83-92.

38. Itoh H, Ueguchi-Tanaka M, Sato Y, Ashikari M, Matsuoka M: The gibberellin signaling pathway is regulated by the appearance and disappearance of SLENDER RICE1 in nuclei. Plant Cell 2002, 14(1):57-70.

39. Bolle C: The role of GRAS proteins in plant signal transduction and development. Planta 2004, 218(5):683-692.

Page 9/20 
40. Daviere JM, Achard P: Gibberellin signaling in plants. Development 2013, 140(6):1147-1151.

41. Sun X, Jones WT, Harvey D, Edwards PJ, Pascal SM, Kirk C, Considine T, Sheerin DJ, Rakonjac J, Oldfield CJ: N-terminal domains of DELLA proteins are intrinsically unstructured in the absence of interaction with GID1/gibberellic acid receptors. J. Biol. Chem. 2010, 285(15):11557-11571.

42. Fu X, Richards DE, Fleck B, Xie D, Burton N, Harberd NP: The Arabidopsis mutant sleepy1gar2-1 protein promotes plant growth by increasing the affinity of the SCFSLY1 E3 ubiquitin ligase for DELLA protein substrates. The Plant Cell 2004, 16(6):1406-1418.

43. Itoh H, Shimada A, Ueguchi-Tanaka M, Kamiya N, Hasegawa Y, Ashikari M, Matsuoka M: Overexpression of a GRAS protein lacking the DELLA domain confers altered gibberellin responses in rice. Plant J. 2005, 44(4):669-679.

44. Blanco-Touriñán N, Serrano-Mislata A, Alabadí D: Regulation of DELLA proteins by post-translational modifications. Plant Cell Physiology 2020, 61(11):1891-1901.

45. Yin JL, Liu MY, Ma DF, Wu JW, Li SL, Zhu YX, Han B: Identification of circular RNAs and their targets during tomato fruit ripening. Postharvest. Biol. Technol. 2018, 136:90-98.

46. Zhou R, Zhu YX, Zhao J, Fang ZW, Wang SP, Yin JL, Chu ZH, Ma DF: Transcriptome-Wide Identification and Characterization of Potato Circular RNAs in Response to Pectobacterium carotovorum Subspecies brasiliense Infection. Int. J. Mol. Sci. 2018, 19(1):71.

47. Blanco-Touriñán N, Legris M, Minguet EG, Costigliolo-Rojas C, Nohales MA, Iniesto E, García-León M, Pacín M, Heucken N, Blomeier T et al: COP1 destabilizes DELLA proteins inArabidopsis. Proceedings of the National Academy of Sciences 2020, 117(24):13792-13799.

48. Zhou M, Chen H, Wei D, Ma H, Lin J: Arabidopsis CBF3 and DELLAs positively regulate each other in response to low temperature. Sci. Rep. 2017, 7(1):113.

49. Hauvermale AL, Tuttle KM, Takebayashi Y, Seo M, Steber CM: Loss of Arabidopsis thaliana Seed Dormancy is Associated with Increased Accumulation of the GID1 GA Hormone Receptors. Plant Cell Physiol. 2014, 56(9):1773-1785.

50. Achard P, Liao LL, Jiang CF, Desnos T, Bartlett J, Fu XD, Harberd NP: DELLAs contribute to plant photomorphogenesis. Plant Physiol. 2007, 143(3):11631172.

51. De Vleesschauwer D, Seifi HS, Filipe O, Haeck A, Huu SN, Demeestere K, Höfte M: The DELLA Protein SLR1 Integrates and Amplifies Salicylic Acid- and Jasmonic Acid-Dependent Innate Immunity in Rice. Plant Physiol. 2016, 170(3):1831-1847.

52. Hou XL, Lee LYC, Xia KF, Yen YY, Yu H: DELLAs Modulate Jasmonate Signaling via Competitive Binding to JAZs. Dev. Cell 2010, 19(6):884-894.

53. Fukao T, Bailey-Serres J: Submergence tolerance conferred by Sub1A is mediated by SLR1 and SLRL1 restriction of gibberellin responses in rice. Proc. Natl. Acad. Sci. U. S. A. 2008, 105(43):16814-16819.

54. Sakuraba Y, Bülbül S, Piao W, Choi G, Paek NC: Arabidopsis EARLY FLOWERING 3 increases salt tolerance by suppressing salt stress response pathways. The Plant Journal 2017, 92(6):1106-1120.

55. Djakovic-Petrovic T, Wit Md, Voesenek LA, Pierik R: DELLA protein function in growth responses to canopy signals. The Plant Journal 2007, 51(1):117126.

56. Robson PR, Whitelam GC, Smith H: Selected components of the shade-avoidance syndrome are displayed in a normal manner in mutants of Arabidopsis thaliana and Brassica rapa deficient in phytochrome B. Plant Physiol. 1993, 102(4):1179-1184.

57. Kebrom TH, Chandler PM, Swain SM, King RW, Richards RA, Spielmeyer W: Inhibition of tiller bud outgrowth in the tin mutant of wheat is associated with precocious internode development. Plant Physiol. 2012, 160(1):308-318.

58. Liao Z, Yu H, Duan J, Yuan K, Yu C, Meng X, Kou L, Chen M, Jing Y, Liu G: SLR1 inhibits MOC1 degradation to coordinate tiller number and plant height in rice. Nature communications 2019, 10(1):1-9.

59. Penfield S, Gilday AD, Halliday KJ, Graham IA: DELLA-mediated cotyledon expansion breaks coat-imposed seed dormancy. Curr. Biol. 2006, 16(23):23662370.

60. An H, Qi XS, Gaynor ML, Hao Y, Gebken SC, Mabry ME, McAlvay AC, Teakle GR, Conant GC, Barker MS et al: Transcriptome and organellar sequencing highlights the complex origin and diversification of allotetraploid Brassica napus. Nature Communications 2019, 10(1):1-12.

61. Jeffares DC, Penkett CJ, Bähler J: Rapidly regulated genes are intron poor. Trends Genet. 2008, 24(8):375-378.

62. Zhou Y, Xu DX, Jia LD, Huang XH, Ma GQ, Wang SX, Zhu MC, Zhang AX, Guan MW, Lu K et al: Genome-Wide Identification and Structural Analysis of bZIP Transcription Factor Genes in Brassica napus. Genes 2017, 8(10):288.

63. Sun TP, Gubler F: Molecular mechanism of gibberellin signaling in plants. Annu. Rev. Plant Biol. 2004, 55:197-223.

64. Nagel R: Gibberellin Signaling in Plants: Entry of a new MicroRNA Player. Plant Physiol. 2020(1):5-6.

65. Wang J, Jian HJ, Wang TY, Wei LJ, Li JN, Li C, Liu LZ: Identification of microRNAs Actively Involved in Fatty Acid Biosynthesis in Developing Brassica napus Seeds Using High-Throughput Sequencing. Frontiers in Plant Science 2016, 7:1570.

66. Achard P, Cheng H, De Grauwe L, Decat J, Schoutteten H, Moritz T, Van Der Straeten D, Peng JR, Harberd NP: Integration of plant responses to environmentally activated phytohormonal signals. Science 2006, 311(5757):91-94.

67. Kwon CT, Paek NC: Gibberellic Acid: A Key Phytohormone for Spikelet Fertility in Rice Grain Production. Int. J. Mol. Sci. 2016, 17(5):794-794.

68. Alvey L, Harberd NP: DELLA proteins: integrators of multiple plant growth regulatory inputs? Physiol. Plant. 2005, 123(2):153-160.

69. Josse EM, Gan YB, Bou-Torrent J, Stewart KL, Gilday AD, Jeffree CE, Vaistij FE, Martinez-Garcia JF, Nagy F, Graham IA et al: A DELLA in Disguise: SPATULA Restrains the Growth of the Developing Arabidopsis Seedling. Plant Cell 2011, 23(4):1337-1351.

70. De Lucas M, Davière J-M, Rodríguez-Falcón M, Pontin M, Iglesias-Pedraz JM, Lorrain S, Fankhauser C, Blázquez MA, Titarenko E, Prat S: A molecular framework for light and gibberellin control of cell elongation. Nature 2008, 451(7177):480-484. 
71. Yang DL, Yao J, Mei CS, Tong XH, Zeng LJ, Li Q, Xiao LT, Sun TP, Li JG, Deng XW et al: Plant hormone jasmonate prioritizes defense over growth by interfering with gibberellin signaling cascade. Proc. Natl. Acad. Sci. U. S. A. 2012, 109(19):E1192-E1200.

72. Wang ZJ, Liu L, Cheng CH, Ren ZY, Xu SM, Li X: GAI Functions in the Plant Response to Dehydration Stress in Arabidopsis thaliana. Int. J. Mol. Sci. 2020, 21(3):819.

73. Claeys H, Skirycz A, Maleux K, Inze D: DELLA Signaling Mediates Stress-Induced Cell Differentiation in Arabidopsis Leaves through Modulation of Anaphase-Promoting Complex/Cyclosome Activity. Plant Physiol. 2012, 159(2):739-+.

74. Ko JH, Yang SH, Han KH: Upregulation of an Arabidopsis RING-H2 gene, XERICO, confers drought tolerance through increased abscisic acid biosynthesis. Plant J. 2006, 47(3):343-355.

75. Zentella R, Zhang ZL, Park M, Thomas SG, Endo A, Murase K, Fleet CM, Jikumaru Y, Nambara E, Kamiya Y et al: Global analysis of DELLA direct targets in early gibberellin signaling in Arabidopsis. Plant Cell 2007, 19(10):3037-3057.

76. Piskurewicz U, Jikumaru Y, Kinoshita N, Nambara E, Kamiya Y, Lopez-Molina L: The Gibberellic Acid Signaling Repressor RGL2 Inhibits Arabidopsis Seed Germination by Stimulating Abscisic Acid Synthesis and ABI5 Activity. Plant Cell 2008, 20(10):2729-2745.

77. Achard P, Renou JP, Berthome R, Harberd NP, Genschik P: Plant DELLAs restrain growth and promote survival of adversity by reducing the levels of reactive oxygen species. Curr. Biol. 2008, 18(9):656-660.

78. Magome H, Yamaguchi S, Hanada A, Kamiya Y, Oda K: The DDF1 transcriptional activator upregulates expression of a gibberellin-deactivating gene, GA20x7, under high-salinity stress in Arabidopsis. The Plant Journal 2008, 56(4):613-626.

79. Chou KC, Shen HB: Plant-mPLoc: A Top-Down Strategy to Augment the Power for Predicting Plant Protein Subcellular Localization. PLoS One 2010, 5(6):e11335.

80. Kumar S, Stecher G, Li M, Knyaz C, Tamura K: MEGA X: Molecular Evolutionary Genetics Analysis across Computing Platforms. Mol. Biol. Evol. 2018, 35(6):1547-1549.

81. Bailey TL, Boden M, Buske FA, Frith M, Grant CE, Clementi L, Ren JY, Li WW, Noble WS: MEME SUITE: tools for motif discovery and searching. Nucleic Acids Res. 2009, 37(suppl_2):W202-W208.

82. Chen $\mathrm{C}$, Chen $\mathrm{H}, \mathrm{He} \mathrm{Y}$, Xia R: TBtools, a toolkit for biologists integrating various biological data handling tools with a user-friendly interface. BioRxiv 2018:289660.

83. Wang YP, Tang HB, DeBarry JD, Tan X, Li JP, Wang XY, Lee TH, Jin HZ, Marler B, Guo H et al: MCScanX: a toolkit for detection and evolutionary analysis of gene synteny and collinearity. Nucleic Acids Res. 2012, 40(7):e49-e49.

84. Stern A, Doron-Faigenboim A, Erez E, Martz E, Bacharach E, Pupko T: Selecton 2007: advanced models for detecting positive and purifying selection using a Bayesian inference approach. Nucleic Acids Res. 2007, 35(suppl_2):W506-W511.

85. Wang D, Zhang Y, Zhang Z, Zhu J, Yu J: KaKs_Calculator 2.0: a toolkit incorporating gamma-series methods and sliding window strategies. Genomics, proteomics bioinformatics. 2010, 8(1):77-80.

86. Wei LJ, Zhu Y, Liu RY, Zhang AX, Zhu MC, Xu W, Lin A, Lu K, Li JN: Genome wide identification and comparative analysis of glutathione transferases (GST) family genes in Brassica napus. Sci. Rep. 2019, 9(1):1-13.

87. Yi X, Zhang ZH, Ling Y, Xu WY, Su Z: PNRD: a plant non-coding RNA database. Nucleic Acids Res. 2015, 43(D1):D982-D989.

88. Lescot M, Dehais P, Thijs G, Marchal K, Moreau Y, Van de Peer Y, Rouze P, Rombauts S: PlantCARE, a database of plant cis-acting regulatory elements and a portal to tools for in silico analysis of promoter sequences. Nucleic Acids Res. 2002, 30(1):325-327.

89. Swift ML: GraphPad prism, data analysis, and scientific graphing. J. Chem. Inf. Comput. Sci. 1997, 37(2):411-412.

90. Xin H, Ni XC, Pan X, Wei L, Min Y, Yu K, Qin LW, Wei H: Comparative Transcriptome Analyses Revealed Conserved and Novel Responses to Cold and Freezing Stress in Brassica napus L. G3-Genes Genomes Genetics 2019, 9(8):2723-2737.

91. Erpei L, Hebi Z, Jinjian Y, Liu X, Huahong H, Muyuan Z, Zaikang T: Genome survey of Chinese fir (Cunninghamia lanceolata): Identification of genomic SSRs and demonstration of their utility in genetic diversity analysis. Sci. Rep. 2020, 10(1).

92. Girard IJ, Tong CB, Becker MG, Mao XY, Huang JY, de Kievit T, Fernando WGD, Liu SY, Belmonte MF: RNA sequencing of Brassica napus reveals cellular redox control of Sclerotinia infection. Journal of Experimental Botany 2017, 68(18):5079-5091.

\section{Tables}

Table 1. Characterization of BnDELLA family proteins 


\begin{tabular}{|c|c|c|c|c|c|c|c|c|c|c|c|c|}
\hline \multirow[t]{2}{*}{ Group } & \multirow[t]{2}{*}{ Gene Name } & \multirow[t]{2}{*}{ Gene LOCUS ID } & \multirow{2}{*}{$\begin{array}{l}\text { Chromosome } \\
\text { Number }\end{array}$} & \multicolumn{3}{|l|}{ Location } & \multicolumn{6}{|c|}{ Protein } \\
\hline & & & & Start & End & Orientation & ORF & AA & $\mathrm{PI}$ & $\mathrm{Mw}(\mathrm{Da})$ & GRAVY & \\
\hline \multirow[t]{4}{*}{ I } & BnA09GAl & BnaA09G0218400ZS & scaffoldA09 & 15333875 & 15336195 & Reverse & 1740 & 579 & 5.23 & 63323.35 & -0.247 & 8 \\
\hline & BnCO9GAl & BnaC09G0254100ZS & scaffoldC09 & 23759020 & 23761368 & Reverse & 1710 & 569 & 5.32 & 62327.38 & -0.232 & 8 \\
\hline & $B n A 06 R G A$ & BnaA06G0409200ZS & scaffoldA06 & 46345733 & 46347952 & Forward & 1722 & 573 & 5.58 & 62511.61 & -0.203 & 8 \\
\hline & $B n C 07 R G A$ & BnaC07G0269400ZS & scaffoldC07 & 41375597 & 41377853 & Reverse & 1734 & 577 & 5.41 & 62782.98 & -0.177 & 8 \\
\hline \multirow[t]{2}{*}{ II } & BnC02RGL 1 & BnaC02G0205300ZS & scaffoldC02 & 17476351 & 17477874 & Forward & 1524 & 507 & 5.37 & 55827.07 & -0.159 & 9 \\
\hline & BnA02RGL 1 & BnaA02G0160500ZS & scaffoldA02 & 9357771 & 9367517 & Forward & 1527 & 508 & 5.94 & 56584.09 & -0.215 & 8 \\
\hline \multirow[t]{4}{*}{ III } & BnA05RGL2 & BnaA05G0486300ZS & scaffoldA05 & 44153415 & 44155599 & Forward & 1635 & 544 & 4.71 & 59184.6 & -0.189 & 8 \\
\hline & $\begin{array}{l}\text { BnA05RGL2- } \\
2\end{array}$ & BnaA05G0485400ZS & scaffoldA05 & 44093973 & 44096162 & Forward & 1641 & 546 & 4.69 & 59456.9 & -0.188 & 8 \\
\hline & BnCO9RGL3 & BnaC09G0489900ZS & scaffoldC09 & 60072165 & 60073742 & Forward & 1578 & 525 & 4.8 & 57604.95 & -0.211 & 8 \\
\hline & BnA10RGL3 & BnaA10G0194400ZS & scaffoldA10 & 21533732 & 21535309 & Forward & 1578 & 525 & 4.78 & 57634.01 & -0.203 & 8 \\
\hline
\end{tabular}

ORF (Open reading frame); Al (Alphetic index); pl (Isoelectric point); GRAVY (Grand average of hydropathicity)

Table 2. bn-miRNA targets BnDELLA genes

\begin{tabular}{|c|c|c|c|c|c|c|c|}
\hline miRNA_Acc. & $\begin{array}{l}\text { Gene } \\
\text { symbol }\end{array}$ & Target_Acc. & $\begin{array}{l}\text { Target } \\
\text { start }\end{array}$ & $\begin{array}{l}\text { Target } \\
\text { end }\end{array}$ & miRNA aligned fragment & $\begin{array}{l}\text { alignment } \\
\text { length }\end{array}$ & e-value \\
\hline bna-miR6029 & $B n C 07 R G A$ & BnaC07G0269400ZS & 590 & 610 & UGGGGUUGUGAUUUCAGGCUU & 21 & 5 \\
\hline bna-miR6031 & BnA09GAl & BnaA09G0218400ZS & 1667 & 1690 & AAGAGGUUCGGAGCGGUUUGAAGC & 24 & 5 \\
\hline \multirow[t]{4}{*}{ bna-miR6036 } & $B n A 09 G A l$ & BnaA09G0218400ZS & 1581 & 1591 & \multirow[t]{4}{*}{ AUAGUACUAGUACUUGCAUGAUCA } & 11 & 5.5 \\
\hline & BnCO9GAl & BnaC09G0254100ZS & 1551 & 1561 & & 11 & 5.4 \\
\hline & $B n A 06 R G A$ & BnaA06G0409200ZS & 1563 & 1573 & & 11 & 5.4 \\
\hline & $B n C O 7 R G A$ & BnaC07G0269400ZS & 566 & 578 & & 13 & 0.35 \\
\hline \multirow[t]{3}{*}{ bna-miR6028 } & BnCO9GAl & BnaC09G0254100ZS & 1635 & 1645 & \multirow[t]{3}{*}{ UGGAGAGUAAGGACAUUCAGA } & 11 & 5.4 \\
\hline & BnA06RGA & BnaA06G0409200ZS & 1647 & 1657 & & 11 & 5.4 \\
\hline & $B n C 07 R G A$ & BnaC07G0269400ZS & 1659 & 1669 & & 11 & 5.4 \\
\hline bna-miR390a & $B n A 06 R G A$ & BnaA06G0409200ZS & 275 & 285 & AAGCUCAGGAGGGAUAGCGCC & 11 & 5.4 \\
\hline bna-miR171f & $B n A 06 R G A$ & BnaA06G0409200ZS & 888 & 898 & UGAUUGAGCCGCGCCAAUAUC & 11 & 5.4 \\
\hline bna-miR156b & $B n C 07 R G A$ & BnaC07G0269400ZS & 108 & 123 & UUGACAGAAGAUAGAGAGCAC & 16 & 1.4 \\
\hline bna-miR168b & $B n C 07 R G A$ & BnaC07G0269400ZS & 1079 & 1089 & UCGCUUGGUGCAGGUCGAGAA & 11 & 5.4 \\
\hline bna-miR160a & $B n C 07 R G A$ & BnaC07G0269400ZS & 1530 & 1540 & UGCCUGGCUCCCUGUAUGCCA & 11 & 5.4 \\
\hline $\begin{array}{l}\text { bna- } \\
\text { miR2111a }\end{array}$ & $B n C 02 R G L 1$ & BnaC02G0205300ZS & 1375 & 1391 & GUCCUCGGGAUGCGGAUUACC & 17 & 0.31 \\
\hline bna-miR172b & BnC02RGL 1 & BnaC02G0205300ZS & 1038 & 1048 & GGAAUCUUGAUGAUGCUGCAU & 11 & 4.8 \\
\hline bna-miR166e & BnC02RGL 1 & BnaC02G0205300ZS & 612 & 623 & UCGGACCAGGCUUCAUUCCCC & 12 & 1.2 \\
\hline bna-miR166a & BnA02RGL 1 & BnaA02G0160500ZS & 1187 & 1198 & UCGGACCAGGCUUCAUUCCCC & 12 & 1.2 \\
\hline bna-miR6030 & BnC02RGL 1 & BnaC02G0205300ZS & 736 & 746 & UCCACCCAUACCAUACAGACCC & 11 & 4.8 \\
\hline bna-miR2111c & BnC02RGL 1 & BnaC02G0205300ZS & 1288 & 1298 & UAAUCUGCAUCCUGGGGUUUA & 11 & 4.8 \\
\hline \multirow[t]{2}{*}{ bna-miR172b } & BnA02RGL 1 & BnaA02G0160500ZS & 1041 & 1051 & \multirow[t]{2}{*}{ GGAAUCUUGAUGAUGCUGCAU } & 11 & 4.8 \\
\hline & BnA05RGL2 & BnaA05G0486300ZS & 745 & 755 & & 11 & 5.1 \\
\hline bna-miR390a & BnA10RGL3 & BnaA10G0194400ZS & 1442 & 1452 & AAGCUCAGGAGGGAUAGCGCC & 11 & 4.9 \\
\hline bna-miR168a & BnCO9RGL3 & BnaC09G0489900ZS & 404 & 414 & AAGCUCAGGAGGGAUAGCGCC & 11 & 4.9 \\
\hline
\end{tabular}




\section{Figures}

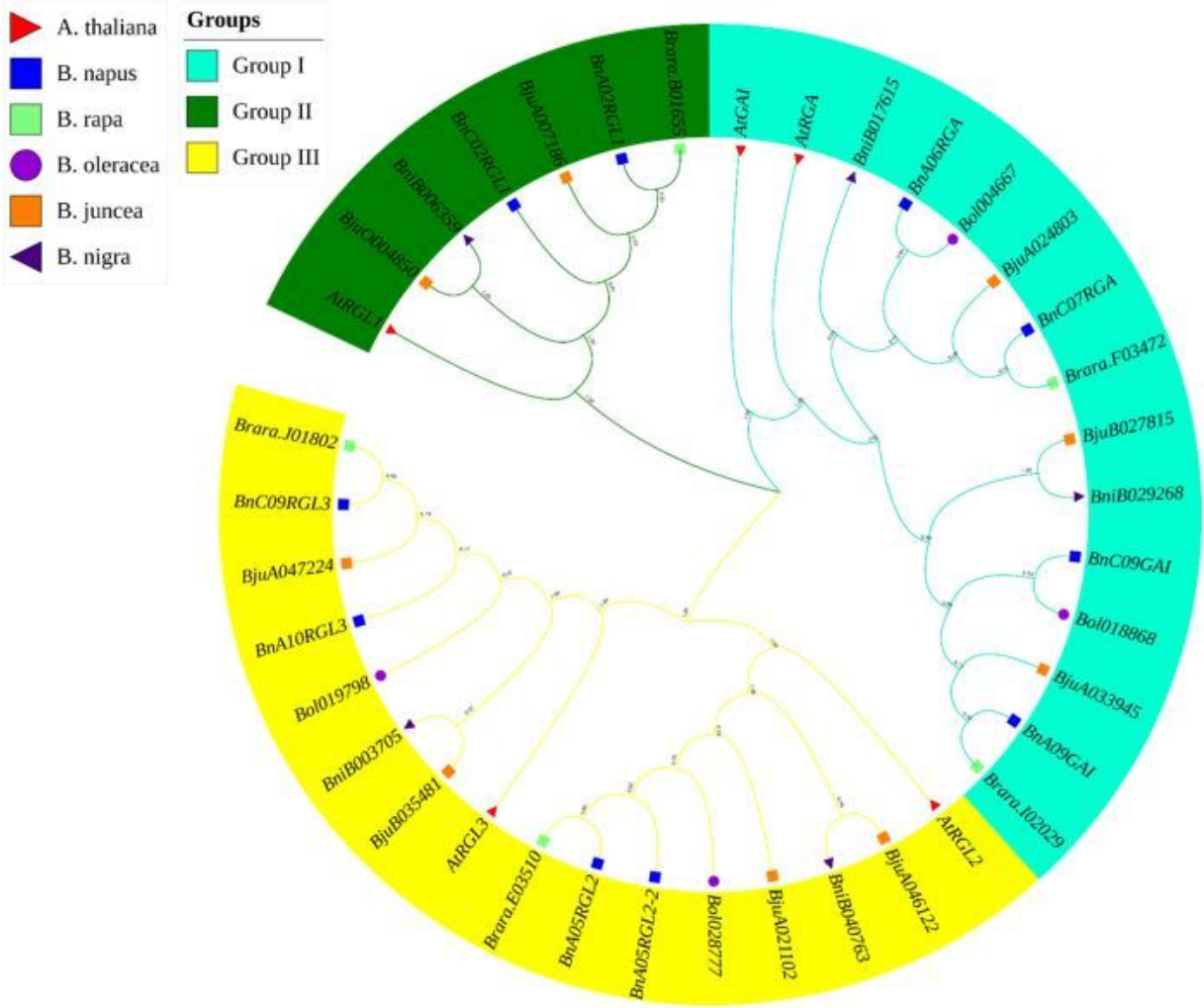

Figure 1

Figure. 1 The cladogram of DELLA proteins from A. thaliana (At:5), B. napus (Bn: 10), B. rapa (Br: 5), B. oleracea (Bo: 4), B. juncea (Bj: 9), B. nigra (Bni: 4) were conducted in MEGA X [80] using the neighbor-joining method, missing data with gaps were eliminated by complete deletion option. The DELLA proteins are cluster into three groups, which are indicated by the different colors. The bootstrap test (1000 replicates) is shown next to the branches. 


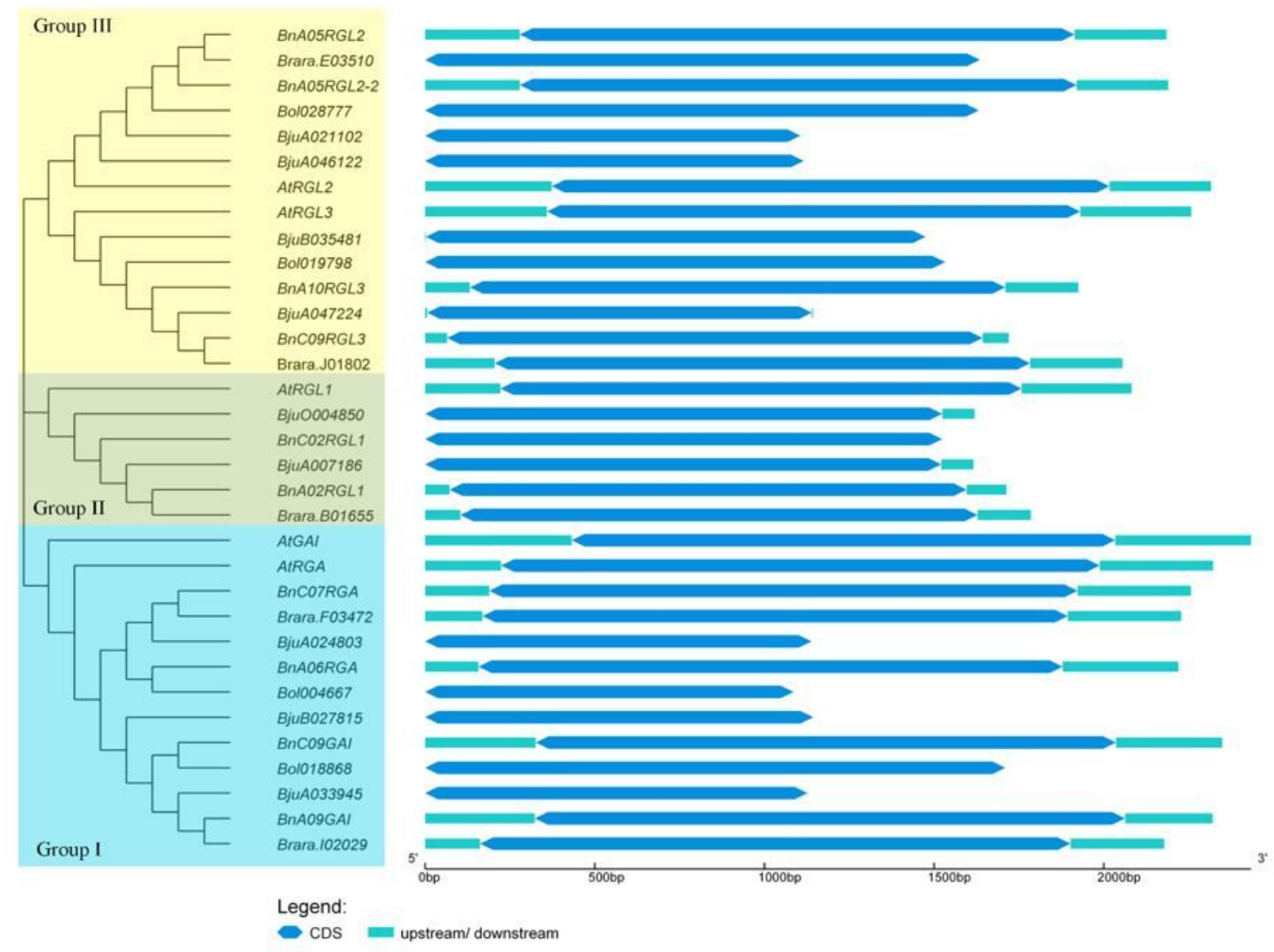

Figure 2

Figure. 2 Exon and intron location of the BnDELLA genes; Blue double-sided wedge represents exon, and upstream/downstream regions are indicated as cyancolored boxes. The scale can estimate the length of the exon at base.

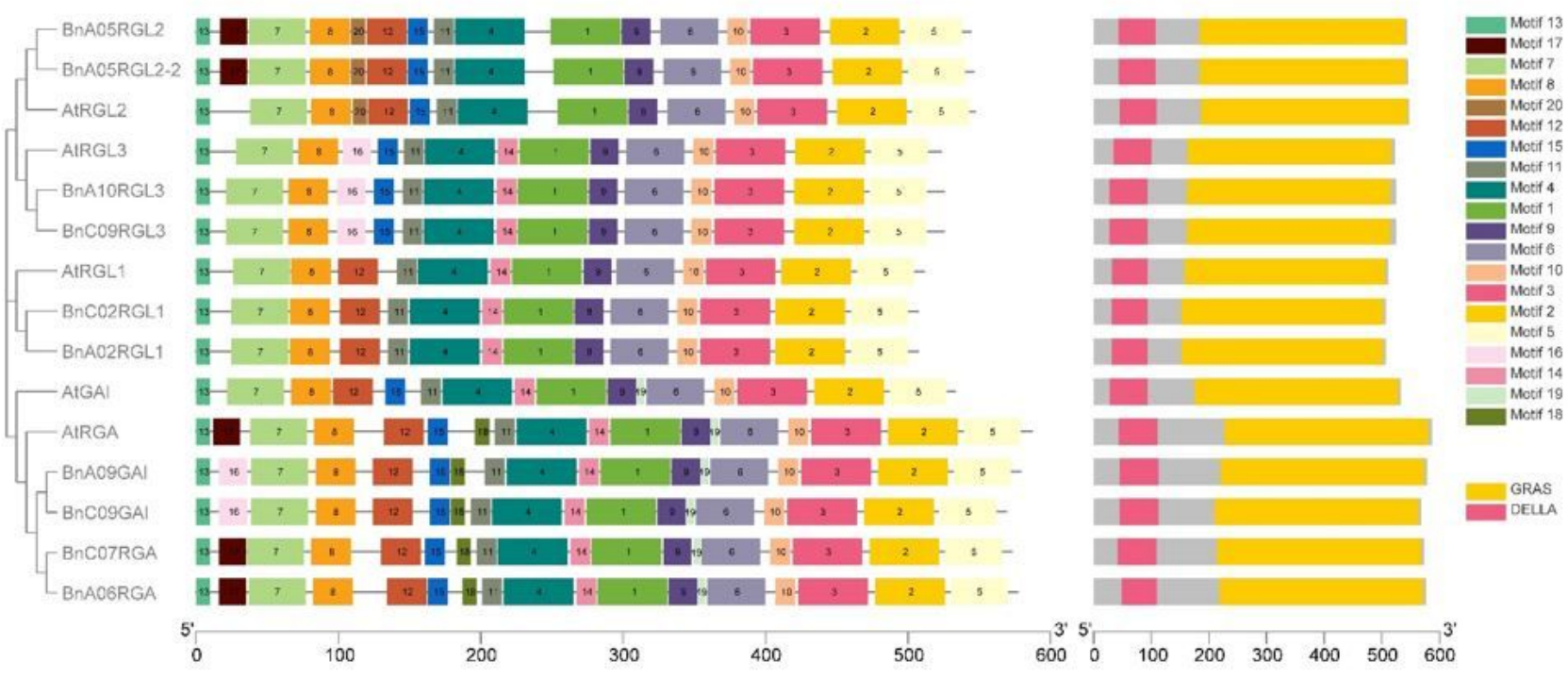

Figure 3

The length of the 20 motifs ranged from 6 to 50 amino acid residues and represent by different colors and numbers, p-values of the motifs on each protein is less than $1 \mathrm{e}-5$ 


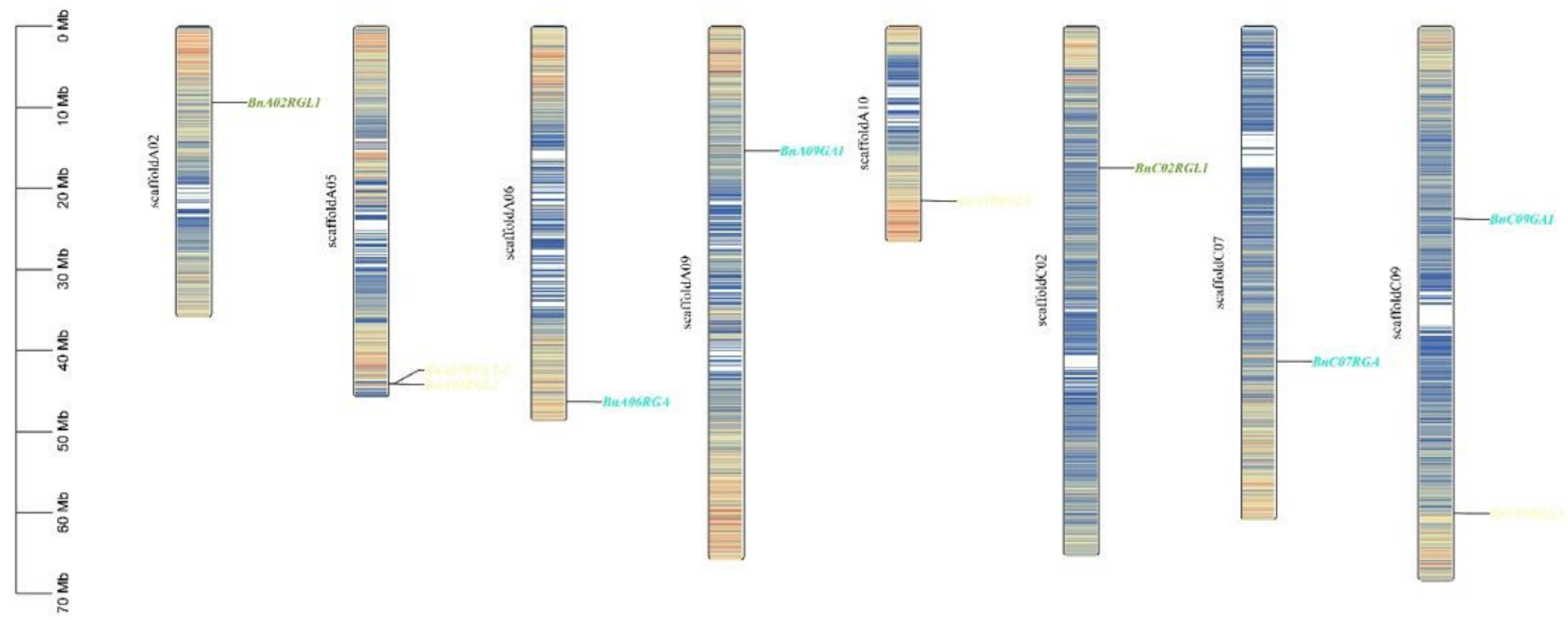

\section{Figure 4}

Schematic representation of DELLA genes distribution on B. napus chromosomes. Chromosome number is indicated on the side of each chromosome.

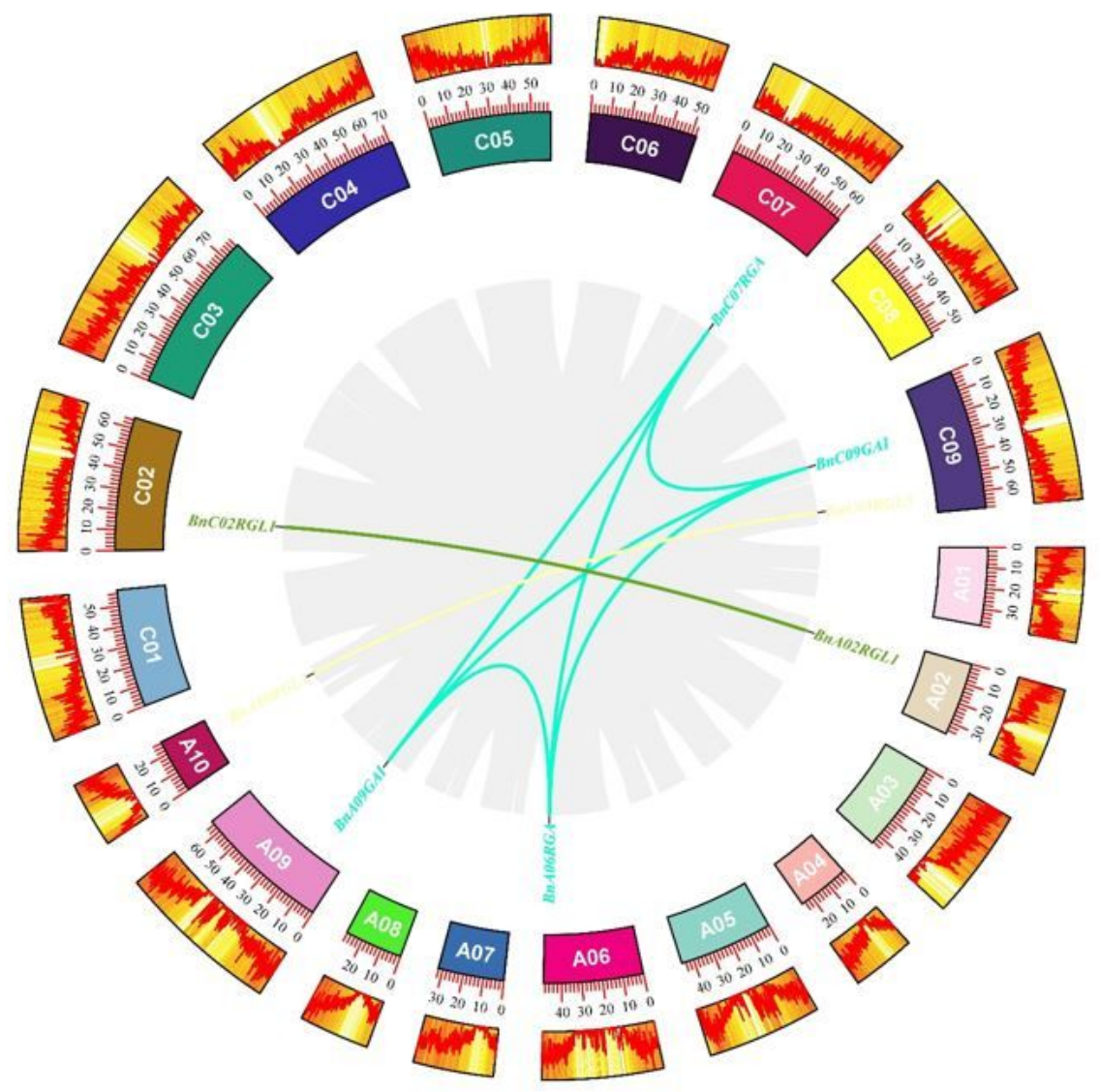

\section{Figure 5}

Synteny analysis of the BnDELLA family in B. napus. Cyan-colored genes belong to group I, and green colored genes belong to group II, yellow-colored genes belong to group III These colored genes indicate duplicated BnDELLA gene pairs, while gray lines represent synteny blocks in the B. napus genome. The distribution density of BnDELLA genes present at the bottom of each chromosome. 


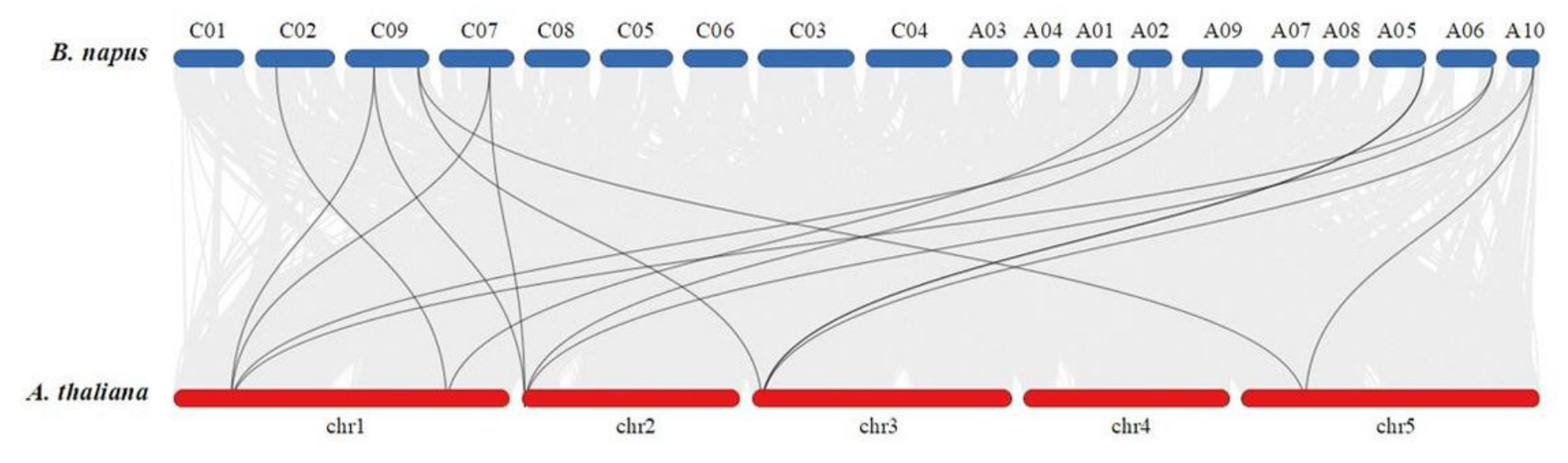

Figure 6

Synteny analysis of DELLA genes between A. thaliana and B. napus. Black lines indicate the syntenic DELLA gene pairs between A. thaliana and B. napus. While in the background, gray line represents collinear blocks.

A

Light Rennosivir

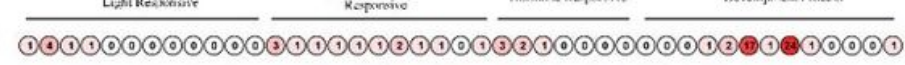

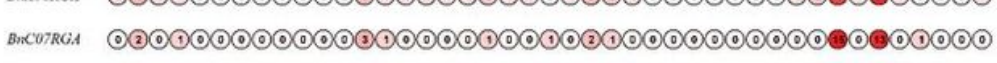

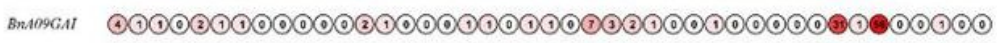

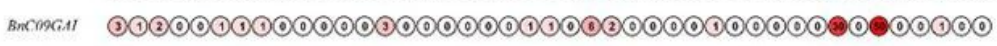

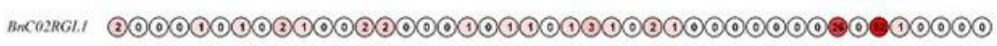

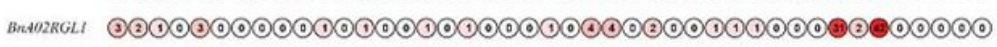

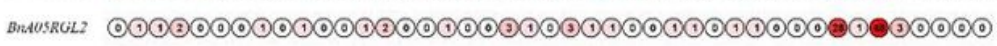

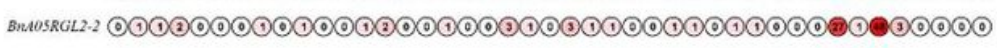

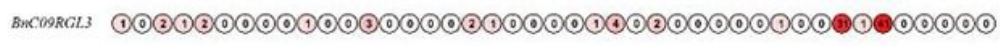

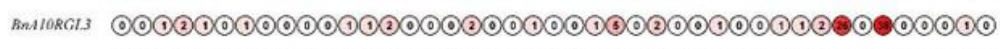

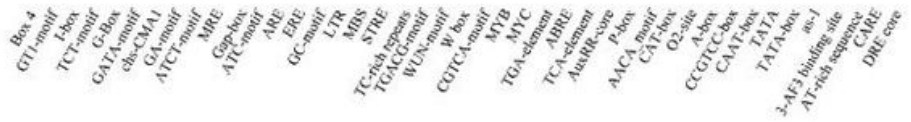
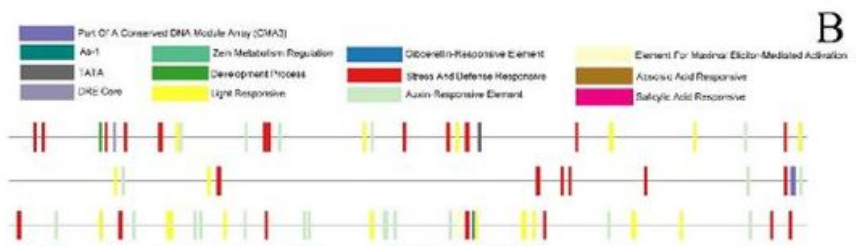

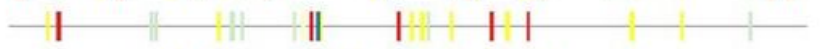
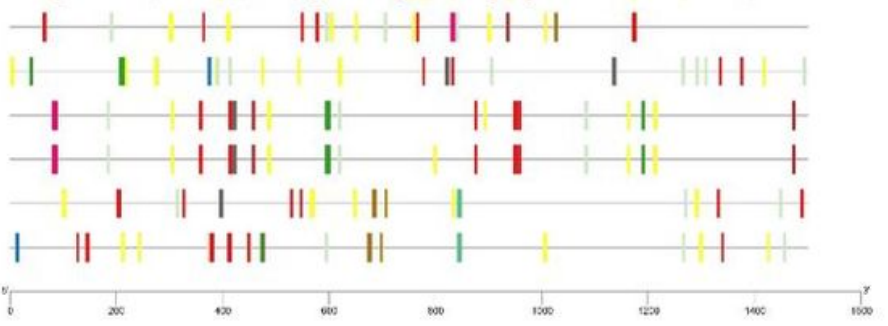

\section{Figure 7}

cis-acting element prediction in the BnDELLA genes. (A) The values in the circle indicated the count of cis-acting element in the promoter of BnDELLA genes (B). The different colored block lines represent the different types and positions of cis-acting elements in each BnDELLA gene. 


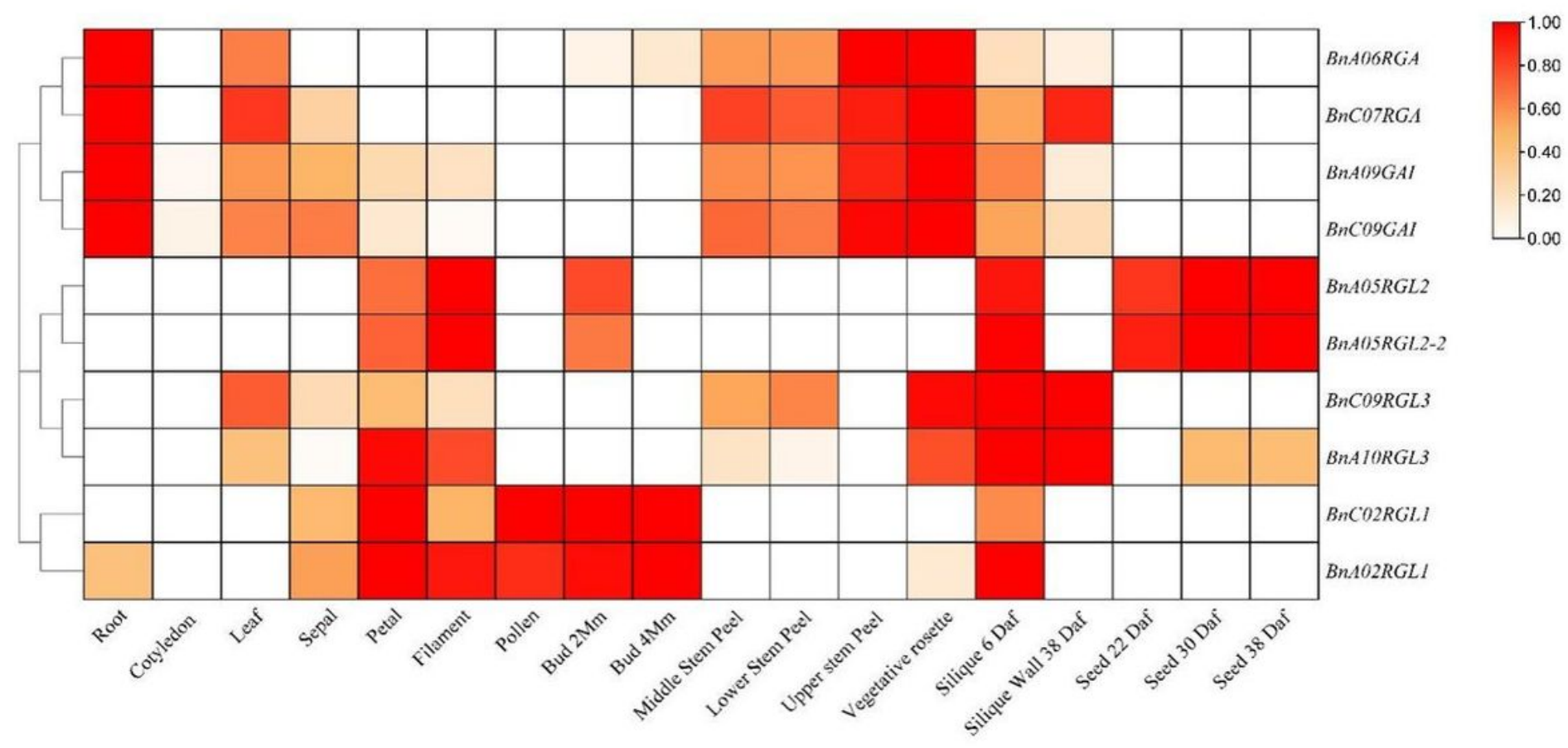

\section{Figure 8}

Heat map showing BnDELLA genes expression in different B. napus tissues. Expression data were analyzed with log2 normalization. Relative expression levels from high (dark-colored) to low (light color) are represented by the color scale bar (listed in Table S4.1). 

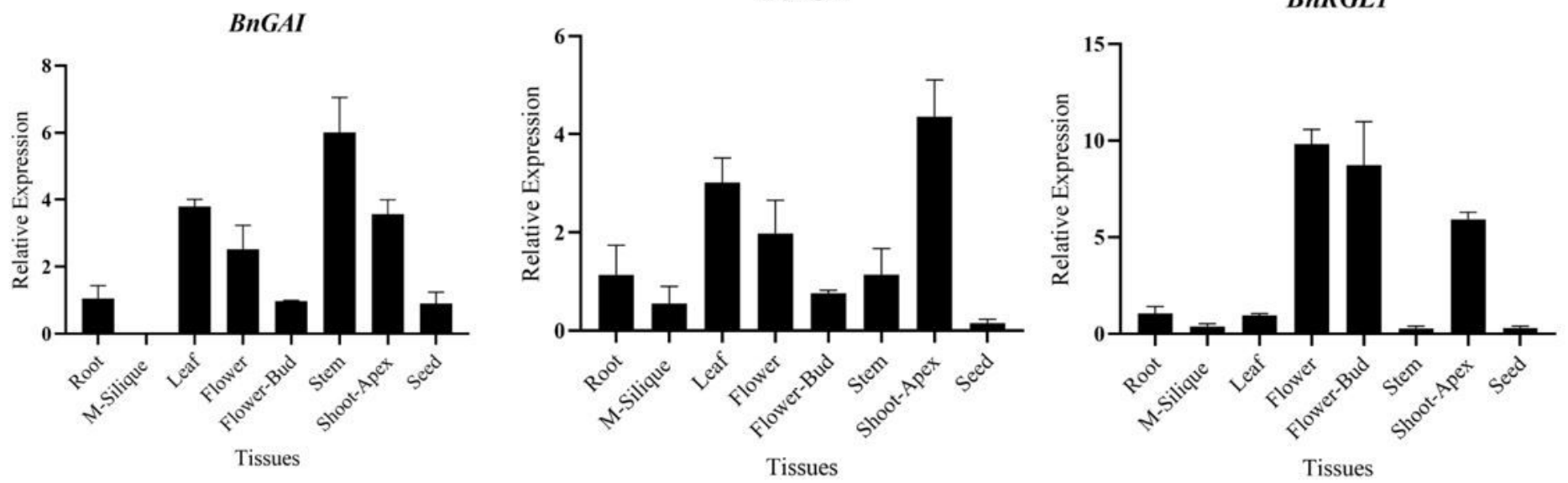

BnRGL2

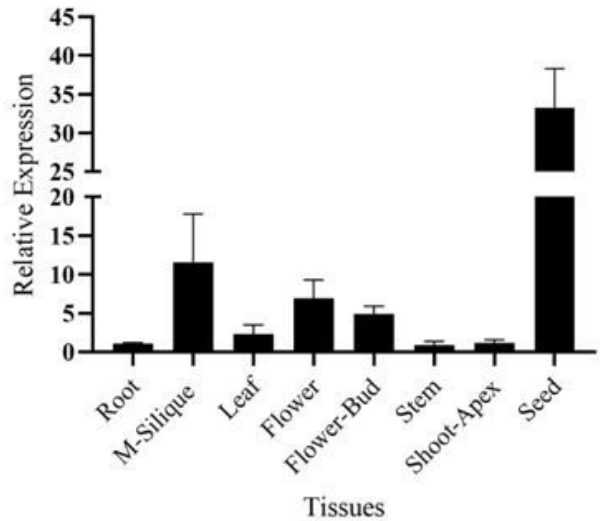

BnRGL3

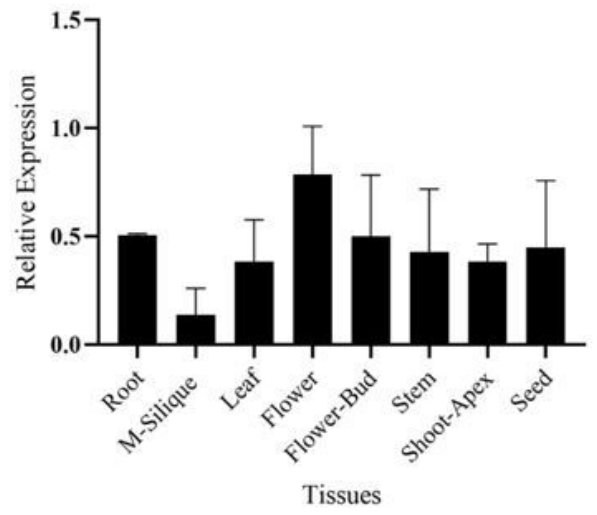

Figure 9

qRT-PCR analysis of the selected BnDELLA genes expression in different organs. The $x$-axis corresponds to different organs, values on the $y$ axis are denoted as the mean \pm SD of three independent replicates (listed in Table S4.2). 

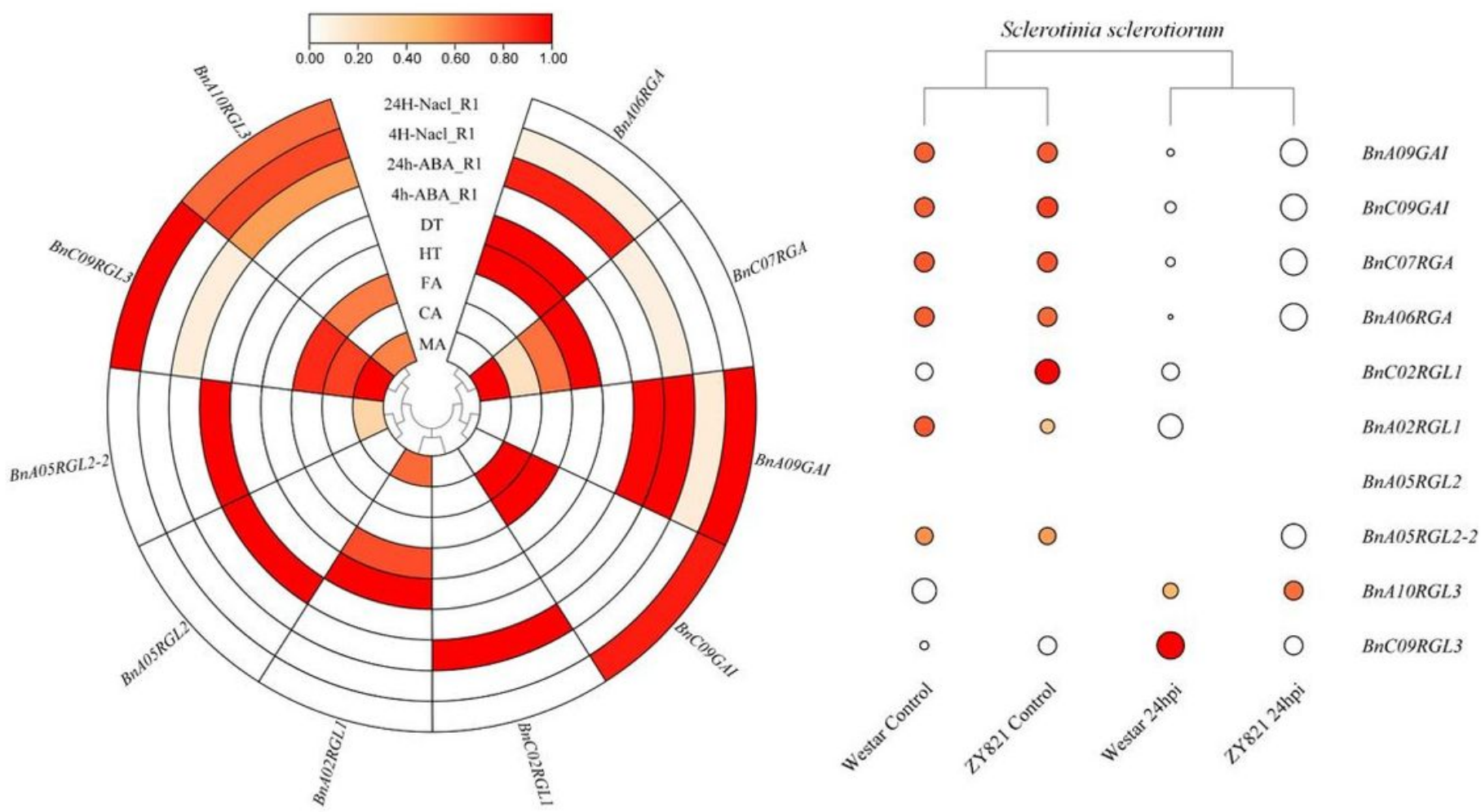

Figure 10

Heat map of the expression profile of BnDELLA genes under different abiotic and biotic conditions including, $\mathrm{MA}$ (Cold shock at chilling $4^{\circ} \mathrm{C}$ and freezing $-4^{\circ} \mathrm{C}$ temperatures), CA (4 degree Celsius $12 \mathrm{~h}$ following cold acclimation 14 days 4 degree Celsius), FA (-4 degree Celsius $12 \mathrm{~h}$ following cold acclimation 14 days 4 degree Celsius), DT (Drought treated), HT (Heat treatment), ABA, NaCl, and Sclerotinia sclerotiorum. The color scale reflects the data of the expression being processed with normalization of log2 (listed in Table S6), and different colors denote different expression levels.

Biological Process

Celluler Component

Molccular Function

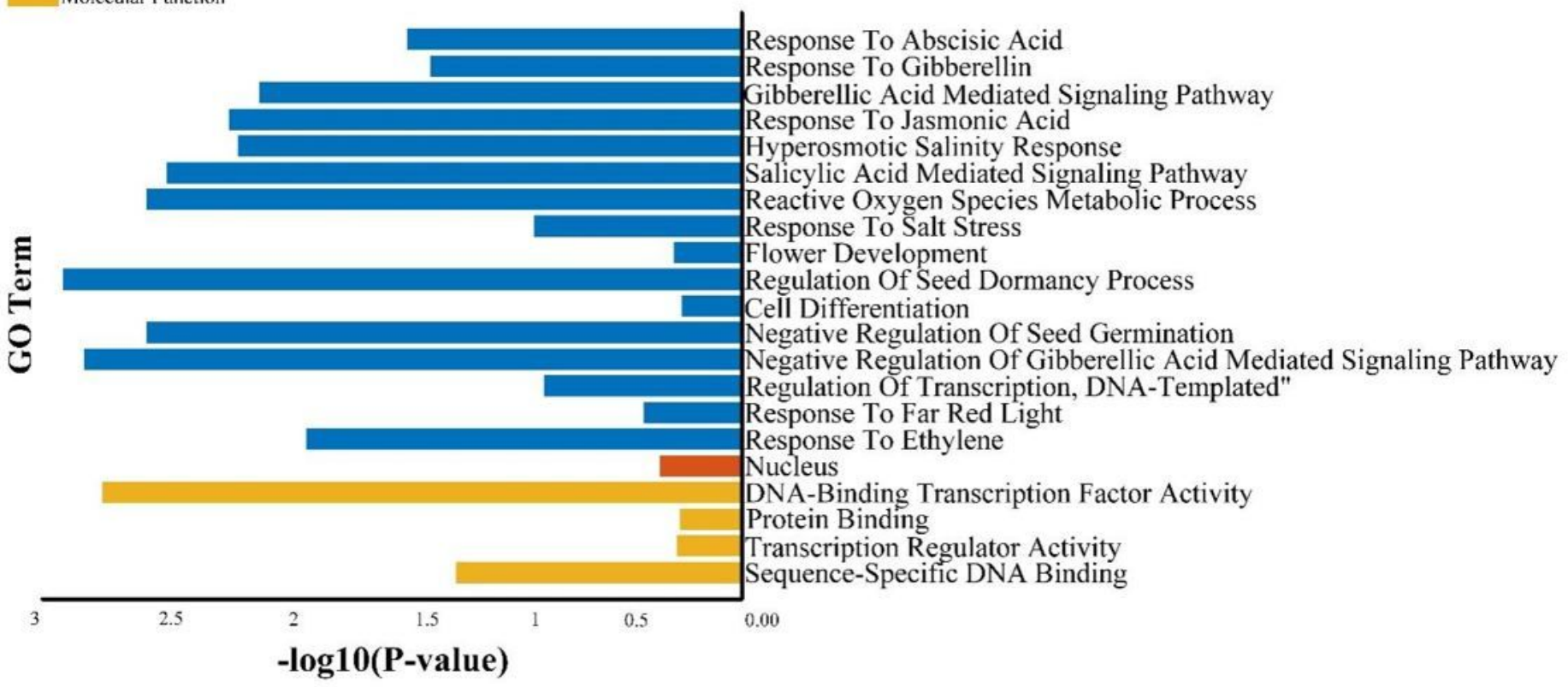

Figure 11 
Gene ontology (GO) analysis. Three categories represent as designated colors, Blue (Biological Process), Red (Cellular Component), Orange (Molecular Function), were used to perform annotation on BnDELLA proteins.

\section{Supplementary Files}

This is a list of supplementary files associated with this preprint. Click to download.

- AdditionalFile1FigureS1.pdf

- AdditionalFile2FigureS2.pdf

- AdditionalFile3FigureS3.pdf

- AdditionalFile4Tables1S8.xlsx 